DRAFT VERSION NOVEMBER 4, 2018

Preprint typeset using LTEX style emulateapj v. 12/16/11

\title{
THE LOCATIONS OF SHORT GAMMA-RAY BURSTS AS EVIDENCE FOR COMPACT OBJECT BINARY PROGENITORS
}

\author{
W. FONG ${ }^{1} \&$ E. BERGER ${ }^{1}$ \\ Draft version November 4, 2018
}

\begin{abstract}
We present a detailed investigation of Hubble Space Telescope rest-frame UV/optical observations of 22 short gamma-ray burst (GRB) host galaxies and sub-galactic environments. Utilizing the high angular resolution and depth of HST we characterize the host galaxy morphologies, measure precise projected physical and hostnormalized offsets between the bursts and host centers, and calculate the locations of the bursts with respect to their host light distributions (rest-frame UV and optical). We calculate a median short GRB projected physical offset of $4.5 \mathrm{kpc}$, about 3.5 times larger than that for long GRBs, and find that $\approx 25 \%$ of short GRBs have offsets of $\gtrsim 10 \mathrm{kpc}$. When compared to their host sizes, the median offset is 1.5 half-light radii $\left(r_{e}\right)$, about 1.5 times larger than the values for long GRBs, core-collapse supernovae, and Type Ia supernovae. In addition, $\approx 20 \%$ of short GRBs having offsets of $\gtrsim 5 r_{e}$, and only $\approx 25 \%$ are located within $1 r_{e}$. We further find that short GRBs severely under-represent their hosts' rest-frame optical and UV light, with $\approx 30-45 \%$ of the bursts located in regions of their host galaxies that have no detectable stellar light, and $\approx 55 \%$ in the regions with no UV light. Therefore, short GRBs do not occur in regions of star formation or even stellar mass. This demonstrates that the progenitor systems of short GRBs must migrate from their birth sites to their eventual explosion sites, a signature of kicks in compact object binary systems. Utilizing the full sample of offsets, we estimate natal kick velocities of $\approx 20-140 \mathrm{~km} \mathrm{~s}^{-1}$. These independent lines of evidence provide the strongest support to date that short GRBs result from the merger of compact object binaries (NS-NS/NS-BH).
\end{abstract}

Keywords: gamma rays: bursts

\section{INTRODUCTION}

The environments of cosmic explosions and their locations within their hosts provide invaluable insight on the nature of their progenitors. For instance, the spatial locations of long gamma-ray bursts (GRBs; $T_{90} \gtrsim 2 \mathrm{~s}$; Kouveliotou et al. 1993) within their exclusively star-forming host galaxies are consistent with the expected distribution for massive stars in exponential disks (Bloom et al. 2002). The result is similar for core-collapse supernovae (SNe; Prieto et al. 2008), which are only found in spiral and irregular galaxies (van den Bergh et al. 2005; Hakobyan et al. 2008; Li et al. 2011), indicative of a young, massive star origin. In contrast, Type Ia $\mathrm{SNe}$ originate in both star-forming and elliptical galaxies (Oemler \& Tinsley 1979, van den Bergh et al. 2005, Mannucci et al. 2005, Li et al. 2011), and their locations do not coincide with star-forming regions (Prieto et al. 2008; Wang et al. 2013), consistent with an evolved progenitor system.

Equally important to the spatial offsets are the locations of these transients with respect to the distribution of the underlying host galaxy light. Using Hubble Space Telescope (HST) observations, it has been shown that long GRBs are concentrated on the brightest ultra-violet (UV) regions of their host galaxies (Fruchter et al. 2006, Svensson et al. 2010), pointing to explosion sites within unusually bright star-forming regions. Similarly, core-collapse SNe tend to explode in bright UV regions within their hosts (Kelly et al. 2008, Svensson et al. 2010). In contrast, Type Ia SNe under-represent their hosts' rest-frame UV light, suggesting that they do not tend to occur in regions of star formation (Kelly et al. 2008, Wang et al. 2013), but may correlate with optical light, suggesting a dependence on stellar mass (Kelly et al. 2008, Wang et al.

\footnotetext{
${ }^{1}$ Harvard-Smithsonian Center for Astrophysics, 60 Garden Street, Cambridge, MA 02138, USA
}

2013) as expected for their white dwarf progenitors.

For short GRBs $\left(T_{90} \lesssim 2 \mathrm{~s}\right)$, several theoretical progenitor systems have been proposed, including NS-NS/NS-BH mergers (Eichler et al. 1989, Narayan et al. 1992), accretioninduced-collapse of a WD or NS (Qin et al. 1998, Levan et al. 2006; Metzger et al. 2008) and magnetar flares (Levan et al. 2006; Chapman et al. 2008). Studies of the short GRB host galaxy demographics have shown that $\approx 1 / 4$ of these events explode in elliptical galaxies with no signs of star formation (Fong et al. 2013), but the majority occur in star-forming galaxies (Berger 2009; Fong et al. 2013). The inferred progenitor ages are $\approx 0.1$-few Gyr (Leibler \& Berger 2010; Fong et al. 2013), pointing to an origin from older stellar populations.

In Fong et al. 2010, we used HST observations of ten short GRB host galaxies to study their sub-galactic environments. For the seven bursts with sub-arcsecond localizations, and thus robust associations to a host galaxy, we constrained their host morphologies, spatial and host-normalized offsets, light distributions, and compared these results to the distributions for long GRBs. We found spatial offsets that are five times greater than those for long GRBs, but with a similar median host-normalized offset of $\approx 1$ half-light radius. In addition, we found preliminary evidence that short GRBs underrepresent their hosts' rest-frame optical and UV light, in stark contrast to long GRBs and core-collapse SNe. Due to the small number of events, the light distribution results were only suggestive, and not statistically significant. Separately, we also used $H S T$ data to study an emerging population of bursts with no obvious coincident host galaxy to optical limits of $\gtrsim 26$ mag and found that these bursts likely have large offsets of 30-100 kpc from their hosts (Berger 2010).

These initial studies demonstrate that HST observations are essential for characterizing the local environments of short 
GRBs, and thus their progenitors. The angular resolution is important in measuring precise offsets and locating the afterglow to sub-pixel precision, critical to analyzing the placement within the host light distributions. In addition, the depth of HST allows for the potential detections of faint coincident host galaxies, surpassing the capabilities of ground-based instuments in the optical and and near-infrared.

Expanding on this initial work, we present here HST observations of 16 additional short GRB host galaxies, 15 of which have sub-arcsecond positions. We describe the data reduction procedures, including photometry, astrometry, surface brightness profile fits, offsets and fractional flux determination in Section 2. We combine the results with those from Fong et al. (2010) and analyze the entire sample of 22 events in Section 3 . Finally, in Section 4 , we consider the implications for the progenitor systems

Throughout the paper we use the standard cosmological parameters, $H_{0}=71 \mathrm{~km} \mathrm{~s}^{-1} \mathrm{Mpc}^{-1}, \Omega_{m}=0.27$, and $\Omega_{\Lambda}=0.73$. All reported magnitudes are corrected for Galactic extinction using dust maps (Schlafly \& Finkbeiner 2011) and are calibrated to the AB magnitude system.

\section{DATA REDUCTION}

\subsection{Sample}

We study HST observations of 16 short GRB host galaxies and environments obtained with the Advanced Camera for Surveys (ACS/WFC) and the infrared and ultravioletvisual channels on the Wide-Field Camera 3 (WFC3/IR and WFC3/UVIS). The data were obtained as part of programs 11669 and 12502 (PI: Fruchter), which targeted all short GRBs with optical positions from April 2007 to January 2010, and the Director's Discretionary Time program 13497 (PI: Tanvir) for GRB 130603B. We combine these public HST data with ground-based observations of optical afterglows to astrometrically locate the burst positions within the host galaxies.

Ten of the 16 bursts have apparent host galaxies based on ground-based imaging (GRBs 070429B, 070707, 070714B, 070724A, 071227, 080905A, 090426A, 090510, 100117A, and 130603B), and all of these except GRB 070707 have spectroscopic redshifts (Cenko et al. 2008, Piranomonte et al. 2008; Berger et al. 2009; D'Avanzo et al. 2009; Graham et al. 2009, Kocevski et al. 2010, Levesque et al. |2010, McBreen et al. 2010; Rowlinson et al. 2010b; Fong et al. 2011; Cucchiara et al. 2013). We note that for GRB 080905A, the host association is less secure (probability of chance coincidence, $\left.P_{c c}(<\delta R) \approx 0.01\right)$ than the remaining bursts with $P_{c c}(<\delta R) \approx$ $10^{-4}-10^{-3}$ (Fong et al. 2013 and Section 3.1), due to the large separation from its claimed host galaxy (Rowlinson et al. 2010b). All of these hosts (except GRB 070429B) have reported near-infrared (NIR) detections or limits (Piranomonte et al. 2008, Berger et al. 2009; Graham et al. 2009; Leibler \& Berger 2010, Levesque et al. 2010; Rowlinson et al. 2010b; Fong et al. 2011; Berger et al. 2013; Tanvir et al. 2013).

The remaining six short GRBs have no known coincident host galaxies to optical limits of $\gtrsim 26 \mathrm{mag}$ from previous ground-based or previous HST observations (GRBs 061201, 070809, 080503, 090305A, 090515, and 091109B; Perley et al. 2008, 2009; Berger 2010; Fong et al. 2010, Rowlinson et al. 2010a, Levan et al. 2009). These events have been termed "host-less", but appear to have host galaxies at separations of $\approx 30-100 \mathrm{kpc}$ with low probability of chance coincidence (Berger 2010).
With the exception of GRB 130603B (Berger et al. 2013, Tanvir et al. 2013), all of the HST observations presented here have not been published in the literature thus far. The results in this work, combined with the HST data from Fong et al. (2010), GRB 080503 (Perley et al. 2009; Berger 2010), and GRB 130603B (Berger et al. 2013, Tanvir et al. 2013), comprise the full available sample of short GRB hosts with $H S T$ observations. Details of the GRB properties and the observations are provided in Table 1.

\subsection{Image Processing}

We retrieved pre-processed images for the 16 short GRBs from the HST archive ${ }^{2}$ We apply distortion corrections and combine the individual exposures using the astrodrizzle package in PyRAF (Gonzaga et. al. 2012). For the ACS images we use pixfrac $=1.0$ and pixscale $=0.05^{\prime \prime}$ pixel $^{-1}$. For the WFC3/IR images, we use the recommended values of pixfrac $=1.0$ and pixscale $=0.0642^{\prime \prime}$ pixel $^{-1}$, half of the native pixel scale, while for the WFC3/UVIS images, we use pixscale $=0.033^{\prime \prime}$ pixel $^{-1}$. The final drizzled images are shown for ten events with established host galaxies (Figure 1), multi-band observations of GRB 080905A (Figure 22 and five events termed as "host-less" (Figure 3).

We obtained public optical/NIR afterglow discovery images for each burst. These images are from the UV-Optical Telescope (UVOT) on-board the Swift satellite, the twin $6.5-\mathrm{m}$ Magellan telescopes, the Gemini-North and South 8-m telescopes, and the 8-m Very Large Telescope (VLT). The telescope and instrument for each afterglow image is listed in Table 2. For the observations from Magellan, Gemini, and VLT, we use standard IRAF tasks to process the data. For GRBs 070429B and 070714B, which have reported UVOT afterglows (Holland et al. 2007; Landsman et al. 2007), we use the uvotimsum task as part of the HEASOFT package to create co-added images for each of the seven UVOT filters.

We confirm the afterglow detection of GRB 070714B in multiple filters (Landsman et al. 2007). For GRB 070429B, the afterglow is reported to be weak $(3.9 \sigma)$ and detectable only by combining the data from all filters at $\delta t \approx 600-$ $2660 \mathrm{~s}$. To assess whether this is a real detection, we combine the UVOT observations in the same manner as described in Holland et al. (2007), but we do not detect any source at the reported position, nor within the XRT error circle. We therefore do not consider the reported afterglow to be real, and use the XRT position (Table 1) in our analysis presented here.

\subsection{Photometry}

We perform aperture photometry for the galaxy with the lowest probability of chance coincidence in each field (see Section 3.1 using standard tasks in IRAF and the tabulated zeropoints for ACS, WFC3/IR and WFC3/UVIS calibrated to the $A B$ magnitude system (Table 1). In addition, for the bursts previously termed as "host-less" (GRBs 061201, 070809, 080503, 090515, and 091109B; Figure 3), we determine the $3 \sigma$ limit at the afterglow position.

We note that for GRB $080905 \mathrm{~A}$, we can only perform photometry for "G2", because "G1", claimed to be the host galaxy by Rowlinson et al. (2010b), is contaminated by foreground saturated stars which we cannot reliably subtract (Figure 2p. The position of GRB 090305A, which was previously reported to have no coincident host to $r \gtrsim 25.6$ mag

\footnotetext{
${ }^{3}$ http://archive.stsci.edu/hst/.
} 
Table 1

HST Observations of Short GRB Host Galaxies

\begin{tabular}{|c|c|c|c|c|c|c|c|c|c|c|}
\hline GRB & $\begin{array}{c}\text { RA } \\
(\mathrm{J} 2000)\end{array}$ & $\begin{array}{c}\text { Dec } \\
(\mathrm{J} 2000)\end{array}$ & $\begin{array}{l}\text { Uncert. } \\
\left({ }^{\prime \prime}\right)\end{array}$ & $z$ & Instrument & Filter & $\begin{array}{l}\text { Date } \\
\text { (UT) }\end{array}$ & $\begin{array}{l}\text { Exp. Time } \\
\text { (s) }\end{array}$ & $\mathrm{AB} \mathrm{mag}^{a}$ & $\begin{array}{c}A_{\lambda} \\
(\mathrm{mag})\end{array}$ \\
\hline 061201 & $22^{\mathrm{h}} 08^{\mathrm{m}} 32.13^{\mathrm{s}}$ & $-74^{\circ} 34^{\prime} 47.05^{\prime \prime}$ & 0.12 & $0.111 ?$ & WFC3/IR & F160W & 2012 Jun 11 & 6397 & $>26.4 / 18.63 \pm 0.01 / 24.46 \pm 0.03^{b}$ & 0.039 \\
\hline \multirow[t]{2}{*}{ 070429B } & $21^{\mathrm{h}} 52^{\mathrm{m}} 03.81^{\mathrm{s}}$ & $-38^{\circ} 49^{\prime} 42.0^{\prime \prime}$ & $1.5^{c}$ & 0.9023 & WFC3/IR & F160W & 2010 Apr 26 & 2797 & $20.59 \pm 0.03$ & 0.012 \\
\hline & & & & & WFC3/UVIS & F475W & 2010 Apr 26 & 2797 & $24.31 \pm 0.20$ & 0.096 \\
\hline \multirow[t]{2}{*}{070707} & $17^{\mathrm{h}} 50^{\mathrm{m}} 58.59^{\mathrm{s}}$ & $-68^{\circ} 55^{\prime} 27.59^{\prime \prime}$ & 0.16 & $<3.6$ & WFC3/IR & F160W & 2010 Jun 24 & 6397 & $26.16 \pm 0.24$ & 0.035 \\
\hline & & & & & ACS & F606W & 2010 Jun 30 & 5644 & $26.68 \pm 0.12$ & 0.177 \\
\hline \multirow[t]{2}{*}{ 070714B } & $03^{\mathrm{h}} 51^{\mathrm{m}} 22.28^{\mathrm{s}}$ & $+28^{\circ} 17^{\prime} 51.75^{\prime \prime}$ & 0.20 & 0.923 & WFC3/IR & F160W & 2009 Aug 16 & 2798 & $22.99 \pm 0.02$ & 0.069 \\
\hline & & & & & WFC3/UVIS & F475W & 2009 Aug 16 & 2698 & $24.89 \pm 0.06$ & 0.467 \\
\hline 070724A & $01^{\mathrm{h}} 51^{\mathrm{m}} 14.10^{\mathrm{s}}$ & $-18^{\circ} 35^{\prime} 39.28^{\prime \prime}$ & 0.08 & 0.4571 & WFC3/IR & F160W & 2011 Oct 3 & 2396 & $19.89 \pm 0.02$ & 0.006 \\
\hline \multirow[t]{2}{*}{070809} & $13^{\mathrm{h}} 35^{\mathrm{m}} 04.55^{\mathrm{s}}$ & $-22^{\circ} 08^{\prime} 30.8^{\prime \prime}$ & 0.40 & $0.473 ?$ & WFC3/IR & F160W & 2010 May 5 & 5597 & $>26.2 / 18.22 \pm 0.01^{d}$ & 0.041 \\
\hline & & & & & ACS & F606W & 2009 Aug 9 & 5150 & $>28.1 / 20.47 \pm 0.03^{d}$ & 0.210 \\
\hline \multirow[t]{2}{*}{071227} & $03^{\mathrm{h}} 52^{\mathrm{m}} 31.25^{\mathrm{s}}$ & $-55^{\circ} 59^{\prime} 02.63^{\prime \prime}$ & 0.22 & 0.381 & WC3/IR & F160W & 2010 Jun 11 & 2797 & $18.73 \pm 0.01$ & 0.006 \\
\hline & & & & & WFC3/UVIS & F438W & 2010 Jun 12 & 2900 & $22.35 \pm 0.05$ & 0.042 \\
\hline 080503 & $19^{\mathrm{h}} 06^{\mathrm{m}} 28.77^{\mathrm{s}}$ & $+68^{\circ} 47^{\prime} 35.32^{\prime \prime}$ & 0.14 & $<4.2$ & WFC3/IR & F160W & 2011 Dec 26 & 5597 & $>26.2 / 25.84 \pm 0.07^{d}$ & 0.028 \\
\hline \multirow[t]{4}{*}{ 080905A } & $19^{\mathrm{h}} 10^{\mathrm{m}} 41.74^{\mathrm{s}}$ & $-18^{\circ} 52^{\prime} 47.44^{\prime \prime}$ & 0.18 & 0.1218 & WFC3/IR & F160W & 2011 Oct 16 & 2397 & & \\
\hline & & & & & WFC3/IR & F160W & 2012 Apr 14 & 2397 & $25.97 \pm 0.11^{e f}$ & 0.014 \\
\hline & & & & & WFC3/UVIS & F814W & 2012 Apr 14 & 2600 & $>27.5^{f}$ & 0.040 \\
\hline & & & & & WFC3/UVIS & F606W & 2012 Apr 14 & 2600 & $27.29 \pm 0.14^{f}$ & 0.066 \\
\hline 090305A & $16^{\mathrm{h}} 07^{\mathrm{m}} 07.59^{\mathrm{s}}$ & $-31^{\circ} 33^{\prime} 22.12^{\prime \prime}$ & 0.20 & $<4.1$ & WFC3/IR & F160W & 2012 Feb 22 & 5597 & $25.20 \pm 0.10$ & 0.092 \\
\hline 090426 & $12^{\mathrm{h}} 36^{\mathrm{m}} 18.05^{\mathrm{s}}$ & $+32^{\circ} 59^{\prime} 09.42^{\prime \prime}$ & 0.12 & 2.609 & WFC3/IR & F160W & 2011 Oct 28 & 2397 & $25.56 \pm 0.07$ & 0.008 \\
\hline 090510 & $22^{\mathrm{h}} 14^{\mathrm{m}} 12.53^{\mathrm{s}}$ & $-26^{\circ} 34^{\prime} 59.0^{\prime \prime}$ & 0.20 & 0.903 & WFC3/IR & F160W & 2011 Oct 11 & 2397 & $21.79 \pm 0.01$ & 0.009 \\
\hline 090515 & $10^{\mathrm{h}} 56^{\mathrm{m}} 36.10^{\mathrm{s}}$ & $+14^{\circ} 26^{\prime} 29.37^{\prime \prime}$ & 0.16 & $0.403 ?$ & WFC3/IR & F160W & 2011 Oct 24 & 5597 & $>26.1 / 18.42 \pm 0.02^{d}$ & 0.001 \\
\hline 091109B & $07^{\mathrm{h}} 30^{\mathrm{m}} 56.61^{\mathrm{s}}$ & $-54^{\circ} 05^{\prime} 23.11^{\prime \prime}$ & 0.16 & $<4.4$ & WFC3/IR & F160W & 2012 Feb 26 & 5596 & $>25.0 / 19.74 \pm 0.03^{d}$ & 0.075 \\
\hline $100117 \mathrm{~A}$ & $00^{\mathrm{h}} 45^{\mathrm{m}} 04.65^{\mathrm{s}}$ & $-01^{\circ} 35^{\prime} 41.99^{\prime \prime}$ & 0.16 & 0.915 & WFC3/IR & F160W & 2011 Sep 29 & 2397 & $21.37 \pm 0.04$ & 0.011 \\
\hline \multirow[t]{2}{*}{ 130603B } & $11^{\mathrm{h}} 28^{\mathrm{m}} 48.17^{\mathrm{s}}$ & $+17^{\circ} 04^{\prime} 18.03^{\prime \prime}$ & 0.09 & 0.3564 & WFC3/IR & F160W & 2013 Jun 13 & 2612 & $19.83 \pm 0.02$ & 0.012 \\
\hline & & & & & ACS & F606W & 2013 Jun 13 & 2216 & $21.08 \pm 0.04$ & 0.057 \\
\hline
\end{tabular}

Note. $-{ }^{a}$ Corrected for Galactic extinction, $A_{\lambda}$ S Schlafly \& Finkbeiner 2011

${ }^{b}$ For GRB 061201, we report the $3 \sigma$ limit on a coincident host galaxy, photometry for "G1" and for "G2", respectively.

${ }^{c} 1 \sigma$ positional error radius from Swift/XRT Goad et al.2007 Evans et al. 2009]

${ }^{d}$ For GRBs $070809,080503,090515$ and $091109 \mathrm{~B}$, we report both the $3 \sigma$ limit on a coincident host galaxy and the magnitude of the galaxy with the lowest probability of chance coincidence.

${ }^{e}$ To attain a higher signal-to-noise ratio, photometry is reported for the 2011 October 16 and 2012 April 14 observations combined.

${ }^{f}$ The position of "G1" is contaminated with saturated stars, so photometry is reported here only for "G2". The F814W limit corresponds to $3 \sigma$.

(Berger 2010), coincides with an extended NIR source with $m_{\mathrm{F} 160 \mathrm{~W}}=25.20 \pm 0.10$ mag which we consider to be the host galaxy (Figure 1, Section 3.1). For GRB 090426A, we consider the host galaxy to be the source directly coincident with the afterglow position (Figure 1. Section 3.1), previously reported to be a "compact knot" within a multi-component host galaxy complex from ground-based observations (Antonelli et al. 2009a; Levesque et al. 2010). For GRB 091109B, for which a host galaxy has not been previously reported, the afterglow position is contaminated by a diffraction spike from a nearby star (Figure 3). We perform photometry at the afterglow position using an aperture radius of $2.5 \theta_{\mathrm{FWHM}}$, and place a $3 \sigma$ limit of $m_{\mathrm{F} 160 \mathrm{~W}} \gtrsim 25 \mathrm{mag}$ on a coincident host galaxy. We note that this NIR limit is substantially shallower than the $3 \sigma$ limit of the image, $\gtrsim 26$ mag. Finally, the F160W observation of GRB 130603B has additional flux from a point source at the optical afterglow position that is not part of the host galaxy (Berger et al. 2013; Tanvir et al. 2013). We perform point spread function (PSF) subtraction of the point source as described in Berger et al. (2013) and calculate the host photometry from the PSF-subtracted image (Figure 1).

The photometry for all events, as well as $3 \sigma$ upper limits for "host-less" events are listed in Table 1 All of our values are consistent with those published in the literature from ground-based data except for GRB 070714B, where we calculate $m_{\mathrm{F} 160 \mathrm{~W}}=22.99 \pm 0.11 \mathrm{mag}$ while the previously published value is $H=23.58 \pm 0.20 \mathrm{mag}$ (Graham et al. 2009).

\subsection{Absolute Astrometry}

To determine the position of each short GRB afterglow, we perform absolute astrometry using point sources in com- mon between the afterglow discovery images and source catalogs (2MASS, SDSS, or USNO-B depending on availability). If the position of the afterglow is contaminated by host galaxy light in the discovery image, we perform image subtraction using the ISIS package (Alard 2000) relative to latetime observations when the afterglow contribution is negligible. We then use SExtractor ${ }^{4}$ to determine the afterglow position in the subtracted image. To determine the astrometric tie from the ground-based image to the catalog, we use the IRAF astrometry routine $\mathrm{C}$ Cmap and find that a secondorder polynomial with six free parameters corresponding to a shift, scale, and rotation in each coordinate, provides a robust tie in all cases with an average $\sigma_{\text {cat } \rightarrow \text { GRB }} \approx 160$ mas. Our afterglow positions are consistent with published positions in all relevant cases, albeit with higher precision. In the cases of GRBs 070809 and 090510 , the afterglow discovery images are not available to us so we use the published positions and uncertainties in our analysis (Perley et al. 2007; Nicuesa Guelbenzu et al. 2012). The absolute afterglow positions and uncertainties are listed in Table 1

\subsection{Relative Astrometry and Offsets}

To determine the position of each GRB relative to its host galaxy, and thus measure precise offsets, we perform relative astrometry by aligning each of the HST observations to the afterglow discovery images. We consider three sources of uncertainty: the afterglow position $\left(\sigma_{\mathrm{GRB}}\right)$, the astrometric tie uncertainty between the ground-based and HST images $\left(\sigma_{\mathrm{GB} \rightarrow \mathrm{HST}}\right)$, and the host galaxy position $\left(\sigma_{\mathrm{gal}}\right)$.

5 http://sextractor.sourceforge.net/ 


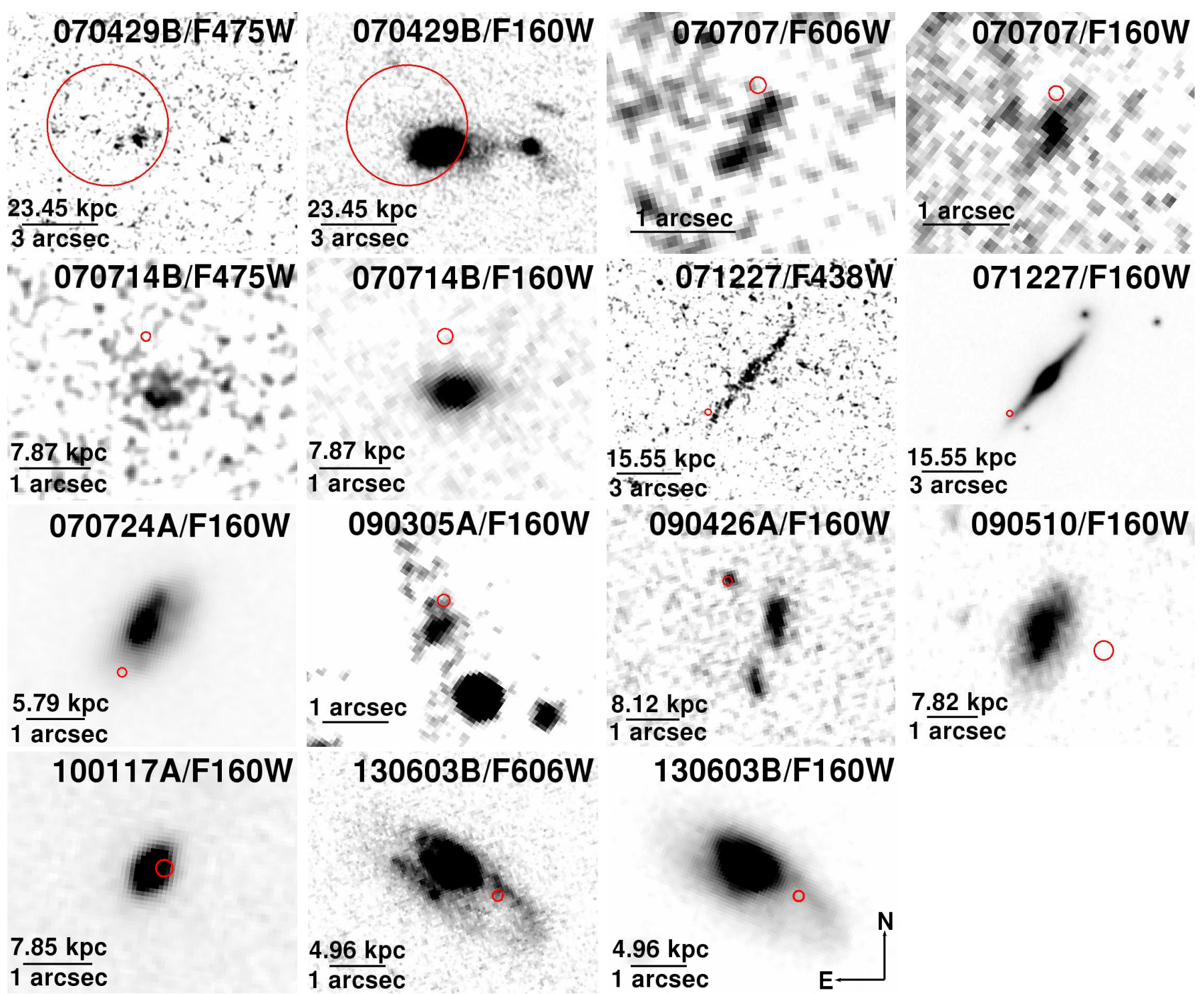

Figure 1. HST observations of ten short GRBs with robust associations to a host galaxy $\left(P_{c c}(<\delta R) \approx 10^{-4}-10^{-3}\right)$ based on previous ground-based observations. The afterglow positions are represented by a $3 \sigma$ error circle in each frame, except for GRBs 070714B and 090510, where the afterglow positional uncertainties are larger, and the circles correspond to $1 \sigma$. For GRB 070429B, the position of the X-ray afterglow from Swift/XRT is shown (red circle; $90 \%$ containment, Goad et al. 2007. Evans et al. 2009). For GRB 130603B/F160W, the image after PSF subtraction of the point source associated with the GRB (see Section 2.3 and Berger et al. 2013) is shown. All images are oriented with North up and East to the left.

We measure $\sigma_{\mathrm{GRB}}$ from each afterglow image, where the centroiding accuracy depends on the size of the PSF and the signal-to-noise ratio of the afterglow detection using SExtractor, and find values of $\sigma_{\mathrm{GRB}} \approx 10-80$ mas $(\mathrm{Ta}-$ ble 2, except in the case of GRB 070429B which has an uncertainty of $1.5^{\prime \prime}(1 \sigma)$ from the Swift/XRT detection of the afterglow. The second source of uncertainty is the astrometric tie between the afterglow and host galaxy HST images $\left(\sigma_{\mathrm{GB} \rightarrow \mathrm{HST}}\right)$, which is determined using the same method described in Section 2.4. We use a range of 5-120 common point sources, depending on the depth of the image and source density and find values of $\sigma_{\mathrm{GB} \rightarrow \mathrm{HST}} \approx 20-110$ mas. The number of astrometric tie objects and resulting RMS values are listed in Table 2. The final source of uncertainty is the centroiding accuracy of the host in the HST images. To determine this uncertainty we again use SExtractor, and find values of $\sigma_{\text {gal }} \approx 1-13$ mas. This is generally the smallest source of uncertainty (Table 2).
For each galaxy/filter combination, we use the afterglow and host position to measure angular offsets, and for the galaxies with known redshifts we also calculate physical offsets (Table 2). We assume $z=1$ for host galaxies without known redshift, taking advantage of the relatively flat value of the angular diameter distance at $z \gtrsim 0.5$. Finally, we use the effective radii, $r_{e}$, determined from surface brightness profile fits (Section 2.6 and Table 3) to calculate host-normalized offsets. The offsets and accompanying combined uncertainties are listed in Table 2. For GRBs 070809 and 090510, where we do not have the afterglow discovery images, we use the published uncertainties of $0.4^{\prime \prime}$ and $0.2^{\prime \prime}$, respectively (Perley et al. 2007; Nicuesa Guelbenzu et al. 2012), which dominate over all other sources of uncertainty.

\subsection{Surface Brightness Profile Fitting}

We use the IRAF/ellipse routine to generate elliptical intensity isophotes and construct one-dimensional radial 


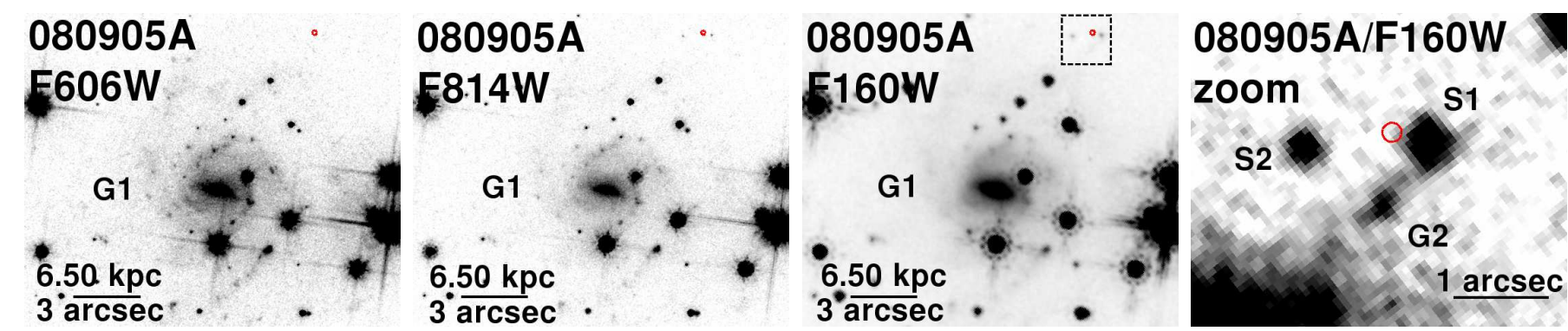

Figure 2. $H S T$ observations of GRB $080905 \mathrm{~A}$ with the optical afterglow position ( $3 \sigma$; red circle) indicated. The face-on spiral galaxy at $z=0.1218$, claimed as the host by Rowlinson et al. $(2010 \mathrm{~b})$, is labeled as "G1" $\left(P_{c c}(<\delta R) \approx 0.01\right)$ in each of the 3 filters, while a zoomed version of the F160W observation shows a new extended source, "G2" $\left(P_{c c}(<\delta R) \approx 0.08\right)$, as well as two sources with stellar PSFs denoted as "S1" and "S2". All images are oriented with North up and East to the left.

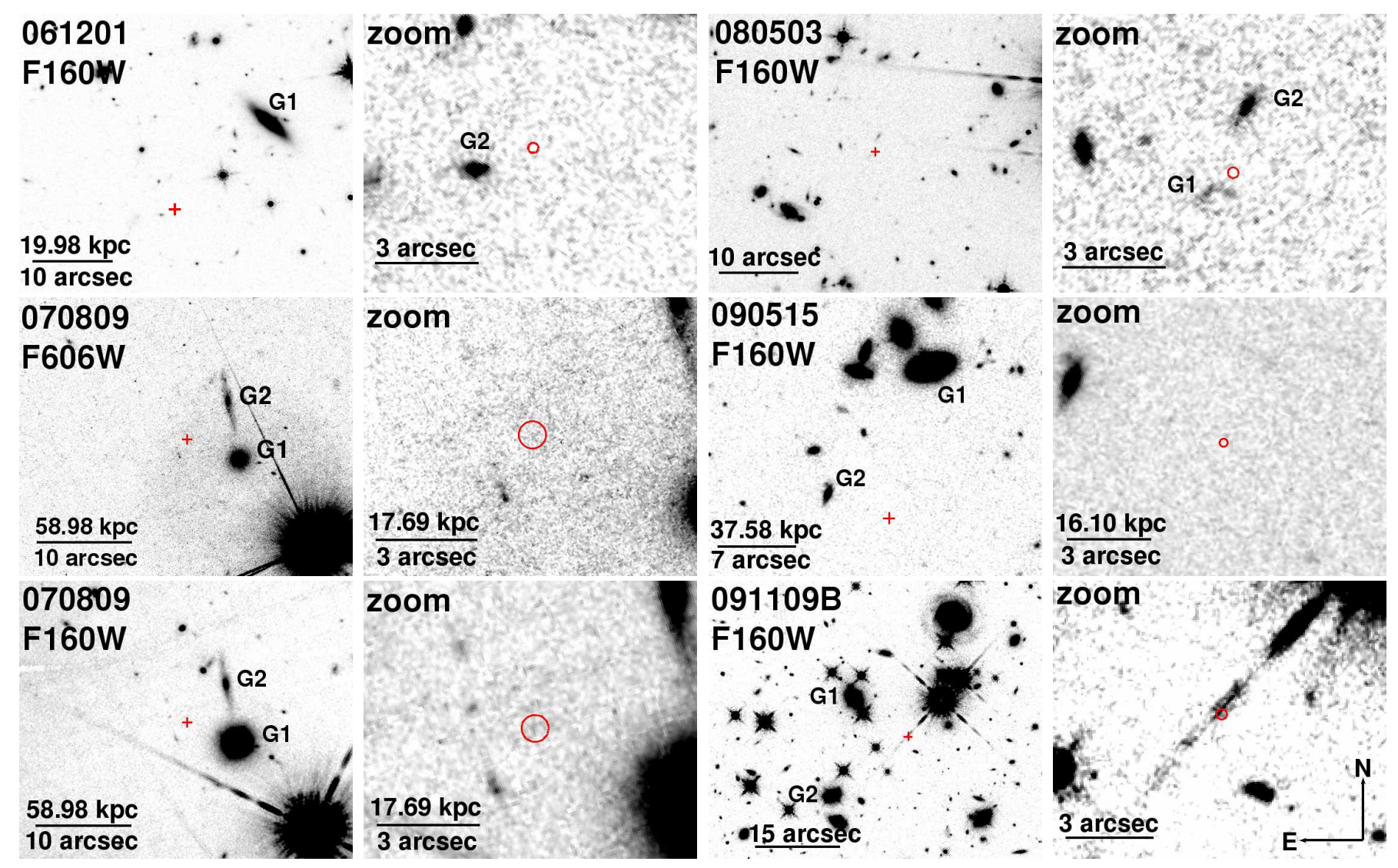

Figure 3. $H S T$ observations of 5 short GRBs with no coincident host galaxy to $m_{160 \mathrm{w}} \gtrsim 26.2 \mathrm{mag}$ ("host-less" bursts). We note that the afterglow position of GRB 091109B is contaminated by a diffraction spike so we place a comparatively shallow limit on a coincident host galaxy of $m_{160 \mathrm{w}} \gtrsim 25 \mathrm{mag}$. The large-scale environments (left) and the $10^{\prime \prime}$ surrounding the afterglow position (right) are shown for each burst. The most probable and second most probable host galaxies from probability of chance coincidence analysis are labeled ("G1" and "G2", respectively). The afterglow positions are shown by the red cross or error circle in each frame. Error circles are $5 \sigma$ in radius except for GRB 070809 , which is $1 \sigma$ because the uncertainty is based on absolute astrometry. Physical scales in kpc are based on the redshift of "G1" for each burst, if known. All images are oriented with North up and East to the left.

surface brightness profiles for each galaxy/filter combination. For each observation, we allow the center, ellipticity, and position angle of each isophote to vary. In two cases (GRB 070707/F606W and GRB 071227/F438W), the isophotal fit does not converge, which can be attributed to the low signal-to-noise ratio of these observations. The surface brightness profiles are displayed in Figure 4

Using a $\chi^{2}$-minimization grid search, we fit each profile with a Sérsic model given by

$$
\Sigma(r)=\Sigma_{e} \exp \left\{-\kappa_{n}\left[\left(r / r_{e}\right)^{1 / n}-1\right]\right\}
$$

where $n$ is the concentration parameter $(n=1$ is equivalent to an exponential disk profile, while $n=4$ is the de Vaucouleurs profile typical of elliptical galaxies), $\kappa_{n} \approx 2 n-1 / 3+$ $4 / 405 n+46 / 25515 n^{2}$ is a constant that depends on $n$ (Ciotti \& Bertin 1999), $r_{e}$ is the effective radius, and $\Sigma_{e}$ is the effective surface brightness in flux units. We convert $\Sigma_{e}$ to units of mag $\operatorname{arcsec}{ }^{-2}$, designated as $\mu_{e}$. In our grid search, $n, r_{e}$, and $\mu_{e}$ are the three free parameters. A single Sérsic component provides an adequate fit $\left(\chi_{\nu}^{2} \approx 0.4-1.5\right)$ for most of the host galaxies. In four cases (GRBs 090510, 090515, $100117 \mathrm{~A}$, and 130603B) a single component fit yields $\chi_{\nu}^{2} \gtrsim 2$. To improve the fit for these cases, we use two separate Sérsic components corresponding to the inner and outer regions 
Table 2

Short GRB Astrometry and Offsets

\begin{tabular}{|c|c|c|c|c|c|c|c|c|c|c|c|c|c|c|}
\hline GRB & Instrument & Filter & $z$ & Reference & No. & $\begin{array}{c}\sigma_{\mathrm{cat} \rightarrow \mathrm{GRB}} \\
\quad(\mathrm{mas})\end{array}$ & $\begin{array}{c}\sigma_{\mathrm{GB} \rightarrow \mathrm{HST}} \\
(\mathrm{mas})\end{array}$ & $\begin{array}{l}\sigma_{\mathrm{GRB}} \\
\text { (mas) }\end{array}$ & $\begin{array}{c}\sigma_{\mathrm{gal}} \\
\text { (mas) }\end{array}$ & $\begin{array}{c}\delta \mathrm{RA} \\
\left({ }^{\prime \prime}\right)\end{array}$ & $\begin{array}{c}\delta \text { Dec } \\
\left({ }^{\prime \prime}\right)\end{array}$ & $\begin{array}{c}\text { Offset } \\
\left({ }^{\prime \prime}\right)\end{array}$ & $\begin{array}{c}\text { Offset } \\
(\mathrm{kpc})\end{array}$ & $\begin{array}{c}\text { Offset } \\
\left(r_{e}\right)\end{array}$ \\
\hline 061201 & VLT/FORS2 & I & $0.111 ?$ & 2MASS & 17 & 115 & $\ldots$ & 17 & & & & & & \\
\hline G1 & WFC3 & F160W & & VLT & 14 & $\ldots$ & 25 & $\cdots$ & 1 & -11.82 & 11.14 & $16.25 \pm 0.03$ & $32.47 \pm 0.06$ & $14.91 \pm 0.03$ \\
\hline G2 & WFC3 & F160W & & VLT & 14 & $\cdots$ & 25 & $\cdots$ & 2 & 1.69 & -0.62 & $1.80 \pm 0.03$ & $14.47 \pm 0.24^{a}$ & $6.43 \pm 0.11$ \\
\hline \multirow[t]{2}{*}{ 070429B } & WFC3 & F160W & 0.902 & USNO-B & 4 & 150 & $\ldots$ & 1460 & 1.0 & $\ldots$ & $\ldots$ & $<1.46$ & $<11.41$ & $<2.25$ \\
\hline & WFC3 & F475W & & USNO-B & 6 & 290 & $\cdots$ & 1460 & 1.0 & $\cdots$ & $\cdots$ & $<1.46$ & $<11.41$ & $\ldots{ }^{b}$ \\
\hline \multirow[t]{3}{*}{070707} & VLT/FORS1 & $R$ & $<3.6$ & 2MASS & 50 & 156 & $\ldots$ & 10 & & & & & & \\
\hline & WFC3 & F160W & & VLT & 76 & $\ldots$ & 26 & $\ldots$ & 4.3 & -0.16 & -0.01 & $0.40 \pm 0.03$ & $3.22 \pm 0.24^{a}$ & $1.11 \pm 0.08$ \\
\hline & ACS & F606W & & VLT & 97 & $\ldots$ & 24 & $\ldots$ & 12.5 & -0.16 & 0.05 & $0.40 \pm 0.03$ & $3.22 \pm 0.24^{a}$ & $\ldots{ }^{b}$ \\
\hline \multirow[t]{3}{*}{ 070714B } & Swift/UVOT & white & 0.923 & 2MASS & 30 & 199 & $\ldots$ & 13 & & & & & & \\
\hline & WFC3 & F160W & & Swift & 6 & $\ldots$ & 110 & $\ldots$ & 1 & -0.40 & -1.50 & $1.55 \pm 0.11$ & $12.21 \pm 0.87$ & $4.56 \pm 0.33$ \\
\hline & UVIS & F475W & & Swift & 5 & $\cdots$ & 66 & $\cdots$ & 7 & -0.40 & -1.50 & $1.55 \pm 0.07$ & $12.21 \pm 0.53$ & $5.55 \pm 0.24$ \\
\hline \multirow[t]{2}{*}{ 070724A } & Gemini-N/GMOS & $i$ & 0.4571 & 2MASS & 6 & 81 & $\cdots$ & 13 & & & & & & \\
\hline & WFC3 & F160W & & Gemini & 32 & $\cdots$ & 22 & $\cdots$ & 1 & -0.48 & 0.81 & $0.94 \pm 0.03$ & $5.46 \pm 0.14$ & $1.50 \pm 0.04$ \\
\hline \multirow[t]{2}{*}{070809} & WFC3 & F160W & $0.473 ?$ & USNO-B & 5 & 234 & $\ldots$ & 400 & 1 & -5.18 & -2.21 & $5.63 \pm 0.46^{c}$ & $33.22 \pm 2.71$ & $9.25 \pm 0.75$ \\
\hline & ACS & F606W & & 2MASS & 7 & 220 & $\cdots$ & 400 & 2 & -5.47 & -2.10 & $5.86 \pm 0.46^{c}$ & $35.58 \pm 2.71$ & $9.29 \pm 0.71$ \\
\hline \multirow[t]{3}{*}{071227} & VLT/FORS2 & $R$ & 0.381 & 2MASS & 11 & 214 & $\ldots$ & 22 & & & & & & \\
\hline & WFC3 & F160W & & VLT & 11 & $\cdots$ & 40 & $\cdots$ & 6 & -2.11 & 2.11 & $2.98 \pm 0.05$ & $15.50 \pm 0.24$ & $3.28 \pm 0.05$ \\
\hline & UVIS & F438W & & VLT & 10 & $\cdots$ & 43 & $\cdots$ & 4 & -2.02 & 1.83 & $2.72 \pm 0.02$ & $14.18 \pm 0.25$ & $\ldots b$ \\
\hline \multirow[t]{2}{*}{080503} & Gemini-N/GMOS & $r$ & $\cdots$ & 2MASS & 22 & 139 & $\cdots$ & 10 & & & & & & \\
\hline & WFC3 & F160W & & Gemini & 58 & $\cdots$ & 29 & $\cdots$ & 9 & 0.72 & -0.53 & $0.90 \pm 0.03^{c}$ & $7.24 \pm 0.24^{a}$ & $3.46 \pm 0.12$ \\
\hline 080905A & VLT/FORS2 & $R$ & 0.1218 & 2MASS & 116 & 159 & $\cdots$ & 78 & & & & & & \\
\hline G1 & WFC3 & F160W & & VLT & 46 & $\ldots$ & 41 & $\ldots$ & 1 & 4.34 & -7.06 & $8.29 \pm 0.08$ & $17.96 \pm 0.19$ & $10.36 \pm 0.10$ \\
\hline G1 & WFC3/UVIS & F814W & & VLT & 61 & $\ldots$ & 32 & $\ldots$ & 1 & 4.30 & -7.07 & $8.28 \pm 0.08$ & $17.92 \pm 0.19$ & $10.35 \pm 0.10$ \\
\hline G1 & WFC3/UVIS & F606W & & VLT & 102 & $\ldots$ & 34 & $\ldots$ & 1 & 4.34 & -7.07 & $8.30 \pm 0.09$ & $17.97 \pm 0.18$ & $10.38 \pm 0.11$ \\
\hline G2 & WFC3 & F160W & & VLT & 46 & $\cdots$ & 41 & $\cdots$ & 13 & 0.07 & -0.69 & $0.69 \pm 0.09$ & $5.55 \pm 0.72^{a}$ & $3.45 \pm 0.45$ \\
\hline G2 & WFC3/UVIS & F606W & & VLT & 102 & $\cdots$ & 34 & $\cdots$ & 7 & 0.07 & -0.79 & $0.79 \pm 0.09$ & $6.35 \pm 0.72^{a}$ & $3.97 \pm 0.43$ \\
\hline \multirow[t]{2}{*}{ 090305A } & Gemini-S/GMOS & $r$ & $<4.1$ & 2MASS & 122 & 198 & $\cdots$ & 6 & & & & & & \\
\hline & WFC3 & F160W & & Gemini & 115 & $\cdots$ & 31 & $\cdots$ & 5 & 0.09 & -0.42 & $0.43 \pm 0.03$ & $3.46 \pm 0.24^{a}$ & $1.19 \pm 0.08$ \\
\hline \multirow[t]{2}{*}{090426} & VLT/FORS2 & $R$ & 2.609 & SDSS & 60 & 120 & $\ldots$ & 10 & & & & & & \\
\hline & WFC3 & F160W & & VLT & 9 & $\cdots$ & 29 & $\cdots$ & 4 & -0.04 & 0.04 & $0.06 \pm 0.03^{d}$ & $0.45 \pm 0.25$ & $0.29 \pm 0.14$ \\
\hline 090510 & WFC3 & F160W & 0.903 & USNO-B & 7 & 370 & $\cdots$ & 200 & 2 & 1.24 & 0.45 & $1.33 \pm 0.37$ & $10.37 \pm 2.89$ & $1.99 \pm 0.39^{e}$ \\
\hline \multirow[t]{2}{*}{090515} & Gemini-N/GMOS & $r$ & $0.403 ?$ & SDSS & 109 & 160 & $\ldots$ & 15 & & & & & & \\
\hline & WFC3 & F160W & & Gemini & 61 & $\cdots$ & 22 & $\cdots$ & 1 & -3.73 & 13.47 & $13.98 \pm 0.03^{c}$ & $75.03 \pm 0.15$ & $15.53 \pm 0.03^{e}$ \\
\hline \multirow[t]{2}{*}{ 091109B } & VLT/FORS2 & $R$ & $\ldots$ & 2MASS & 20 & 154 & $\ldots$ & 28 & & & & & & \\
\hline & WFC3 & F160W & & VLT & 39 & & 21 & $\cdots$ & 1 & 9.53 & 6.79 & $11.70 \pm 0.03^{c}$ & $94.07 \pm 0.24$ & $\cdots$ \\
\hline \multirow[t]{2}{*}{ 100117A } & Gemini-N/GMOS & $r$ & 0.915 & SDSS & 95 & 154 & $\cdots$ & 26 & & & & & & \\
\hline & WFC3 & F160W & & Gemini & 21 & $\cdots$ & 33 & $\cdots$ & 1 & 0.16 & 0.03 & $0.17 \pm 0.04$ & $1.32 \pm 0.33$ & $0.57 \pm 0.13^{e}$ \\
\hline \multirow[t]{3}{*}{ 130603B } & Magellan/IMACS & $r$ & 0.3564 & SDSS & 17 & 85 & $\ldots$ & 10 & & & & & & \\
\hline & WFC3 & F160W & & Magellan & 12 & $\ldots$ & 34 & $\ldots$ & 1 & 0.93 & 0.48 & $1.05 \pm 0.04$ & $5.21 \pm 0.17$ & $1.05 \pm 0.04^{e}$ \\
\hline & ACS & F606W & & Magellan & 9 & $\ldots$ & 34 & $\ldots$ & 1 & 0.93 & 0.58 & $1.09 \pm 0.04$ & $5.41 \pm 0.17$ & $1.36 \pm 0.05^{e}$ \\
\hline
\end{tabular}

Note. $-{ }^{a}$ We assume $z=1$ to calculate these projected physical offsets.

${ }^{b}$ The $r_{e}$ measurement is highly uncertain due to the low signal-to-noise ratio of the observation.

${ }^{c}$ Offsets are calculated for the galaxy with the lowest probability of chance coincidence.

${ }^{d}$ The offset is calculated relative to the source in direct coincidence with the burst position (Figure 1 .

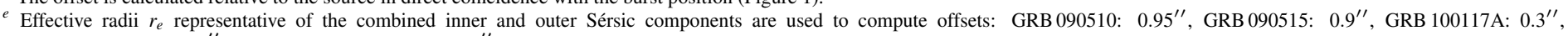
GRB 130603B/F160W: $1^{\prime \prime}$ and GRB 130603B/F606W: $0.8^{\prime \prime}$.

of each galaxy. The resulting values for $n, r_{e}$ and $\mu_{e}$ are listed in Table 3. We do not perform a surface brightness fit for GRB 080905A/“G1" since it is contaminated by saturated foreground sources (Figure 2), but given the prominent spiral structure, the morphology of this host is likely characterized by a disk profile with $n \sim 1$, and we estimate the size of the bulge component to be $\approx 1.8^{\prime \prime}(\approx 3.9 \mathrm{kpc})$. The surface brightness profiles and resulting models are shown in Figure 4

\subsection{Host Light Distributions}

To determine the brightness of the galaxy at the GRB location relative to the host light distribution, we follow the methodology of Fruchter et al. (2006), Kelly et al. (2008), and Fong et al. (2010), and calculate for each galaxy image the fraction of total light in pixels fainter than the afterglow position ("fractional flux"; Table 4). Eleven bursts have differential astrometric positions of better than one pixel (Table 2). If the afterglow position spans multiple pixels, we take the av- 

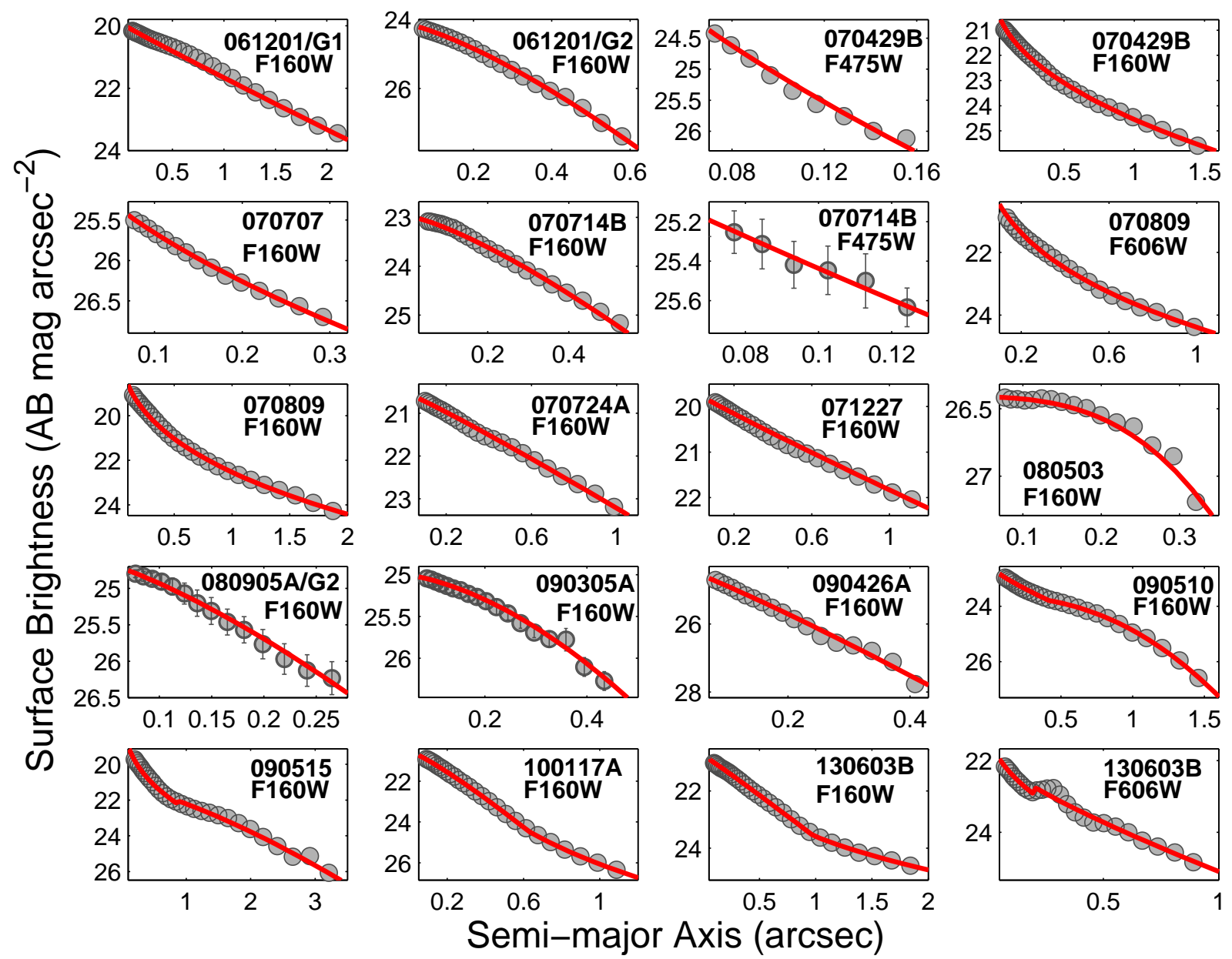

Figure 4. Radial surface brightness profiles as determined from IRAF/el lipse (grey circles) and the corresponding best-fit Sérsic models (red line) for 16 host galaxies. For GRBs 070809, 080503, and 090515, the surface brightness profile for the most probable host galaxy is shown while for GRB 061201, the profiles for both "G1" and "G2" are shown. For GRB 080905A, the profile is shown for the extended source closest to the afterglow position (Source "G2" in Figure 1).

erage brightness among those pixels to be the representative flux of the afterglow position. For each image, we create an intensity histogram of a region centered on the host galaxy and determine a $1 \sigma$ cut-off level for the host by fitting a Gaussian profile to the sky brightness distribution (equivalent to a signal-to-noise ratio cut-off of 1). We then plot the pixel flux distribution above the appropriate cut-off level for a region surrounding the host, and determine the fraction of light in pixels fainter than the afterglow pixel (see Table 4).

For GRBs 071227/F160W and 130603B/F606W, we mask sources contaminating the position of the galaxy and set these pixels to the brightness level of the surrounding pixels. We note that for GRB 080905A, we are unable to explicitly calculate the fractional flux with respect to "G1" due to the presence of saturated stars, while for GRB 061201 we cannot make a unique host association (Section 3.1). However, both of these bursts have afterglow locations at the level of the sky background, and thus have fractional flux values of zero regardless of their host associations. Table 4 also includes the values for seven bursts from Fong et al. (2010).

\section{ANALYSIS}

\subsection{Probabilities of Chance Coincidence}

To assess the probability that each of the bursts originated from a coincident galaxy or from another galaxy in the field, we perform aperture photmetry for galaxies within the $H S T$ field of view, discarding noticeably fainter galaxies with increasing distance from the burst since these objects will have a lower probability of being the host galaxy. We then calculate the probability of chance coincidence, $P_{c c}(<\delta R)$ for each galaxy based on the distance from the burst position $(\delta R)$ and apparent magnitude $(m)$ (c.f., Bloom et al. 2002, Berger 2010). For bursts at offsets of $<1 r_{e}$ (GRBs 090426 and 100117A), we use the effective size of the galaxy, $\delta R=r_{e}$, while for the remaining cases, we take $\delta R$ to be the projected distance between the burst and candidate host center. For the bursts with previously established hosts (Figure 1), we find $P_{c c}(<\delta R) \approx 10^{-4}-10^{-3}$, consistent with ground-based results (Fong et al. 2013); the next probable hosts have values at least one order of magnitude greater, with $P_{c c}(<\delta R) \approx 0.02-0.30$. The lowest value of 0.02 is for GRB 090426A, which has two galaxies within $1^{\prime \prime}$ of the optical afterglow position, in addition to the source at the GRB position. From previous groundbased observations, these sources were considered to comprise a single host galaxy complex (Antonelli et al. 2009b; Levesque et al. 2010). However, given the lack of apparent interaction between these sources in the HST image (see Figure 1 and the comparatively low value of $P_{c c}(<\delta R) \approx 10^{-4}$ for the source at the afterglow position, we consider the latter to be the host galaxy. For GRB 080905A/“G1” (Figure 2), we are unable to accurately determine the brightness in any of the filters due to the presence of foreground saturated stars, but 
Table 3

Short GRB Host Galaxy Morphological Properties

\begin{tabular}{|c|c|c|c|c|c|}
\hline GRB & Filter & $n$ & $\begin{array}{l}r_{e} \\
\left({ }^{\prime \prime}\right)\end{array}$ & $\begin{array}{c}r_{e} \\
(\mathrm{kpc})\end{array}$ & $\begin{array}{c}\mu_{e} \\
\left(\mathrm{AB} \mathrm{mag} \operatorname{arcsec}^{-2}\right)\end{array}$ \\
\hline 061201/G1 & $160 \mathrm{~W}$ & 1.03 & 1.09 & 2.18 & 21.86 \\
\hline 061201/G2 & $160 \mathrm{~W}$ & 0.73 & 0.28 & $2.25^{a}$ & 25.34 \\
\hline \multirow[t]{2}{*}{ 070429B } & $160 \mathrm{~W}$ & 2.15 & 0.65 & 5.08 & 23.61 \\
\hline & $475 \mathrm{~W}$ & 1.54 & $0.08^{b}$ & 0.62 & 24.69 \\
\hline 070707 & $160 \mathrm{~W}$ & 0.97 & 0.36 & $2.89^{a}$ & 27.01 \\
\hline \multirow[t]{2}{*}{ 070714B } & $160 \mathrm{~W}$ & 0.76 & 0.34 & 2.68 & 24.35 \\
\hline & $475 \mathrm{~W}$ & 1.18 & 0.28 & 2.20 & 27.17 \\
\hline 070724A & $160 \mathrm{~W}$ & 0.92 & 0.63 & 3.64 & 22.16 \\
\hline \multirow{2}{*}{070809} & $160 \mathrm{~W}$ & 3.03 & 0.61 & 3.59 & 21.47 \\
\hline & $606 \mathrm{~W}$ & 3.38 & 0.65 & 3.83 & 23.64 \\
\hline 071227 & $160 \mathrm{~W}$ & 1.05 & 0.91 & 4.72 & 21.64 \\
\hline 080503 & $160 \mathrm{~W}$ & 0.32 & 0.26 & $2.09^{a}$ & 26.81 \\
\hline $080905 \mathrm{~A} / \mathrm{G} 1^{c}$ & $160 \mathrm{~W}$ & $\approx 1$ & $\approx 1.8$ & $\approx 3.9$ & $\ldots$ \\
\hline 080905A/G2 & $160 \mathrm{~W}$ & 0.70 & 0.20 & $1.60^{a}$ & 25.72 \\
\hline $090305 \mathrm{~A}$ & $160 \mathrm{~W}$ & 0.57 & 0.36 & $2.89^{a}$ & 25.96 \\
\hline 090426A & $160 \mathrm{~W}$ & 0.89 & 0.21 & 1.70 & 25.78 \\
\hline $090510\left(a<0.4^{\prime \prime}\right)$ & 160W & 1.27 & 0.93 & 7.27 & 24.92 \\
\hline $090510\left(a>0.4^{\prime \prime}\right)$ & $160 \mathrm{~W}$ & 0.44 & 0.74 & 5.79 & 24.26 \\
\hline $090515\left(a<0.85^{\prime \prime}\right)$ & $160 \mathrm{~W}$ & 2.95 & 0.79 & 4.24 & 22.02 \\
\hline $090515\left(a>0.85^{\prime \prime}\right)$ & $160 \mathrm{~W}$ & 0.73 & 1.19 & 6.39 & 22.47 \\
\hline $100117 \mathrm{~A}\left(a<0.6^{\prime \prime}\right)$ & $160 \mathrm{~W}$ & 0.86 & 0.28 & 2.20 & 22.04 \\
\hline 100117A $\left(a>0.6^{\prime \prime}\right)$ & $160 \mathrm{~W}$ & 4.95 & 0.07 & 0.55 & 18.66 \\
\hline 130603B $\left(a<1^{\prime \prime}\right)$ & $160 \mathrm{~W}$ & 0.96 & 0.62 & 3.07 & 22.51 \\
\hline $130603 \mathrm{~B}\left(a>1^{\prime \prime}\right)$ & $160 \mathrm{~W}$ & 3.81 & 3.25 & 2.02 & 25.71 \\
\hline $130603 \mathrm{~B}\left(a<0.2^{\prime \prime}\right)$ & $606 \mathrm{~W}$ & 1.98 & 0.79 & 3.92 & 24.97 \\
\hline 130603B $\left(a>0.2^{\prime \prime}\right)$ & $606 \mathrm{~W}$ & 1.29 & 0.68 & 3.37 & 24.29 \\
\hline
\end{tabular}

Note. $-{ }^{a}$ Calculated assuming $z=1$.

$b$ Due to the low signal-to-noise ratio of this observation, this measurement likely corresponds to a smaller region within the galaxy, and not the entire galaxy itself.

$c$ Although we cannot perform a surface brightness profile fit for GRB 080905A/“G1", these parameters are estimated from the apparent morphology and effective radius of the bulge component.

using $R \sim 18$ mag determined from Rowlinson et al. (2010b) and our offset of 8.3 ", we find that "G1" has $P_{c c}(<\delta R) \approx 0.01$, while "G2" has $P_{c c}(<\delta R) \approx 0.08$. Therefore, "G1" is the more likely host galaxy, although this case is less clear than the other previously established host associations at small offsets.

For bursts with no obvious host galaxy at small offsets from previous ground-based or HST observations (Figure 3), termed "host-less" (GRBs 061201, 070809, 080503, 090305A, 090515: Berger 2010, 091109B: Levan et al. 2009 ), we calculate a range of probabilities, $P_{c c}(<\delta R) \approx$ $6 \times 10^{-3}-0.08$. The associations are most robust for GRBs 070809 and 090305A with the most probable hosts at offsets of $5.7^{\prime \prime}\left(P_{c c}(<\delta R) \approx 6 \times 10^{-3}\right)$ and $0.43^{\prime \prime}\left(P_{c c}(<\delta R) \approx\right.$ $\left.7 \times 10^{-3}\right)$, respectively. The host association for GRB 070809 is the same as that made in Berger (2010), although here we calculate a lower probability of chance coincidence by a factor of three. We note that the extended source we associate with GRB 090305A was not detected in ground-based observations to $r \gtrsim 25.6$ mag (Berger 2010). Due to the low probability of chance coincidence and the lack of more likely host galaxy candidates in the HST observation, we consider this to be the host galaxy. We calculate moderate probabilities of $\approx 0.05$ for GRBs 080503 and 090515 , with the most probable hosts at offsets of $0.90^{\prime \prime}$ and $14^{\prime \prime}$, respectively. These associations are the same as those previously published (Berger 2010; ;erley et al. 2009) but the probabilities of chance coincidence are lower by a factor of two in this work.

The associations are more ambiguous for GRBs 061201
Table 4

Short GRB Fractional Flux

\begin{tabular}{|c|c|c|c|}
\hline GRB & Instrument & Filter & $1 \sigma$ Fractional Flux \\
\hline \multirow[t]{2}{*}{$050709^{a}$} & WFPC2 & F450W & 0 \\
\hline & ACS & F814W & 0.09 \\
\hline \multirow[t]{2}{*}{$050724^{a}$} & WFPC2 & F450W & 0.03 \\
\hline & WFPC2 & F814W & 0.33 \\
\hline \multirow[t]{2}{*}{$051221^{a}$} & WFPC2 & F555W & 0.54 \\
\hline & WFPC2 & F814W & 0.65 \\
\hline $060121^{a}$ & ACS & F606W & 0.41 \\
\hline \multirow[t]{2}{*}{$060313^{a}$} & ACS & F475W & 0.04 \\
\hline & $\mathrm{ACS}$ & F775W & 0 \\
\hline \multirow[t]{2}{*}{$061006^{a}$} & WFPC2 & F555W & 0.56 \\
\hline & ACS & F814W & 0.63 \\
\hline \multirow[t]{3}{*}{$061201^{a b}$} & ACS & F606W & 0 \\
\hline & ACS & F814W & 0 \\
\hline & WFC3/IR & F160W & 0 \\
\hline \multirow[t]{2}{*}{070707} & WFC3/IR & F160W & 0 \\
\hline & ACS & F606W & 0 \\
\hline \multirow[t]{2}{*}{ 070714B } & WFC3/IR & F160W & 0 \\
\hline & WFC3/UVIS & F475W & 0 \\
\hline 070724A & WFC3/IR & F160W & 0.23 \\
\hline \multirow[t]{2}{*}{$070809^{b}$} & WFC3/IR & F160W & 0 \\
\hline & ACS & F606W & 0 \\
\hline \multirow[t]{2}{*}{071227} & WFC3/IR & F160W & 0.19 \\
\hline & WFC3/UVIS & F438W & 0 \\
\hline $080503^{b}$ & WFC3/IR & F160W & 0 \\
\hline \multirow[t]{3}{*}{ 080905A } & WFC3/IR & F160W & 0 \\
\hline & WFC3/UVIS & F814W & 0 \\
\hline & WFC3/UVIS & F606W & 0 \\
\hline 090305A & WFC3/IR & F160W & 0.30 \\
\hline 090426 & WFC3/IR & F160W & 0.82 \\
\hline 090510 & WFC3/IR & F160W & 0 \\
\hline $090515^{b}$ & WFC3/IR & F160W & 0 \\
\hline $100117 \mathrm{~A}$ & WFC3/IR & F160W & 0.54 \\
\hline \multirow[t]{2}{*}{ 130603B } & WFC3/IR & F160W & 0.27 \\
\hline & ACS/606W & F606W & 0.35 \\
\hline
\end{tabular}

Note. - Fraction of host galaxy light in pixels fainter than the GRB position.

${ }^{a}$ From Fong et al. (2010).

${ }^{b}$ No coincident host to the depth of available HST imaging.

and 091109B. For GRB 061201, the two most probable host galaxies ("G1" and "G2"; Figure 3) have offsets of 16.3" and $1.8^{\prime \prime}$, respectively, and both have $P_{c c}(<\delta R) \approx 0.07$ so the host association is inconclusive. However, "G1" is at a relatively low redshift of $z=0.111$ (Stratta et al. 2007) while "G2" is likely at $z \approx 1$; thus the physical offsets are $\gtrsim 15 \mathrm{kpc}$ in both cases (Table 2). For GRB 091109B, the position is contaminated by a diffraction spike, but if there is no coincident host galaxy at $\gtrsim 25 \mathrm{mag}$, the most probable host has $m_{\mathrm{F} 160 \mathrm{~W}} \approx 19.8 \mathrm{mag}$ at an offset of $11.7^{\prime \prime}$, yielding $P_{c c}(<\delta R) \approx 0.08$. We note that $H S T$ imaging at a different rotation angle will be essential in determining whether this 
burst originates from a host galaxy at $\lesssim 1^{\prime \prime}$ separation or from a galaxy at a larger offset. Due to the uncertainty of the associations for GRBs 061201 and 091109B, we do not include these bursts in our subsequent offset analysis.

Overall, these probability of chance coincidence results agree with those in the literature (Perley et al. 2009; Berger 2010, Rowlinson et al. 2010b), and provide deep NIR limits of $\gtrsim 26.2$ mag for bursts which lack hosts at $\delta R \lesssim$ few arcsec. We discuss the possibility that such bursts originated from galaxies fainter than the detection threshold of the HST observations (and demonstrate that this is unlikely) in more detail in Section 4

\subsection{Morphological Properties}

Using the results from the radial surface brightness profiles (Figure 4), we classify the short GRB hosts in terms of their morphological parameters: Sérsic value, $n$, and effective size, $r_{e}$. We find two elliptical galaxies, the hosts of GRBs 070809 and 090515 , with $n \approx 3.0-3.4$ while the remaining galaxies have disk-like morphologies with $n \approx 0.3-2.1$ (Table 3). We note that GRB 100117A exhibits a complex morphology in the NIR, with Sérsic indices of $n \approx 0.9$ and $\approx 5$ for its inner and outer regions, respectively, although it is spectroscopically classified as an early-type galaxy with a stellar population age of $\approx 1-2 \mathrm{Gyr}$ and no evidence for star formation activity (Fong et al. 2011). GRB 130603B, which is a star-forming galaxy with SFR $\gtrsim 1.3 M_{\odot} \mathrm{yr}^{-1}$ (Cucchiara et al. 2013), has an inner component in the NIR with $n \approx 1$ and a broad outer component with $n \approx 3.8$. This host galaxy has an irregular, asymmetric morphology in the optical band with excess flux at radial distances of $a \approx 0.2-0.4^{\prime \prime}$ in the surface brightness profile (Figure 4 ) and Sérsic components with $n \approx 2$ and 1.3 .

The effective radii range from $\approx 0.2-1.2^{\prime \prime}$ with a median size of $0.36^{\prime \prime}$. We note that the signal-to-noise ratio of the GRB $070429 \mathrm{~B} / \mathrm{F} 475 \mathrm{~W}$ observation is low and the $r_{e}$ measurement likely corresponds to a smaller region within the galaxy, and not the entire galaxy. For the host galaxies that require two Sérsic components, we use the radius which encloses half of the flux as the effective size when computing the host-normalized offsets. These values are 0.95" (GRB 090510), 0.9" (GRB 090515), $0.3^{\prime \prime}$ (GRB 100117A), $1^{\prime \prime}$ (GRB 130603B/F160W) and $0.8^{\prime \prime}$ (GRB 130603B/F606W). For the short GRBs with known redshifts, the median physical size is about $r_{e} \approx 3.6 \mathrm{kpc}$. The smallest hosts are GRBs 070714B and 100117A while the largest are GRBs 090510 and 090515 . The median value for this sample is the same as the value of $3.5 \mathrm{kpc}$ reported in Fong et al. (2010) for a preliminary sample of hosts. Compared to the long GRB median host galaxy size of $1.7 \mathrm{kpc}$ (Wainwright et al.2007), short GRB host galaxies are twice as large. This is consistent with their larger luminosities (Berger 2009) and stellar masses (Leibler \& Berger 2010).

\subsection{Offsets}

To study the locations of short GRBs with respect to their host galaxies, we first consider the distribution of projected angular offsets. The range of angular offsets is $\approx 0.1-14^{\prime \prime}$ with GRB 090426 as the smallest offset and GRB 090515 as the largest. From the angular offsets, we calculate projected

\footnotetext{
${ }^{7}$ Calculated from the galaxy in direct coincidence with the optical afterglow position, using $z=2.609$ as determined from afterglow spectroscopy Antonelli et al. 2009a, Levesque et al.|2010).
}

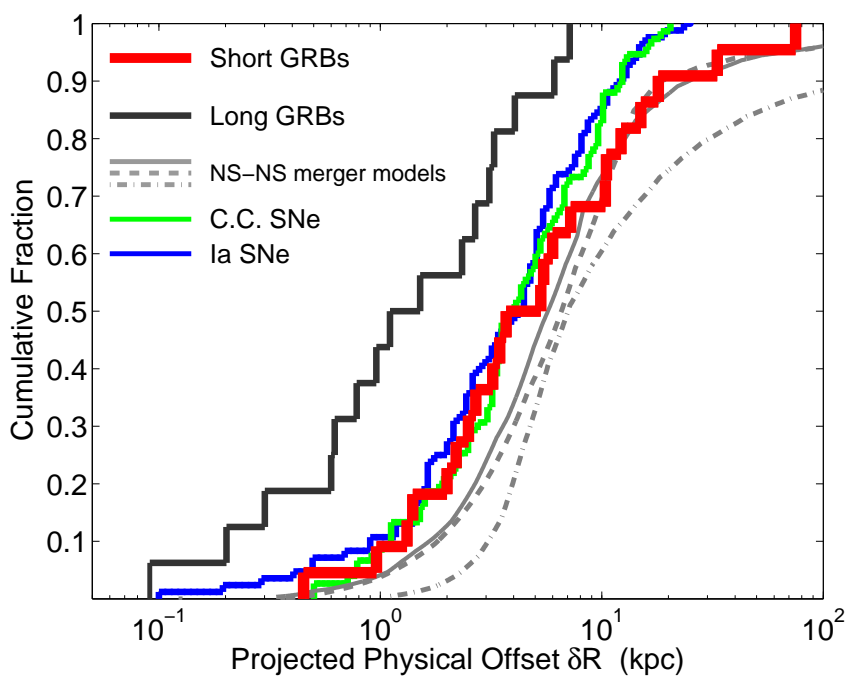

Figure 5. Cumulative distribution of projected physical offsets for 22 short GRBs with sub-arcsecond positions (red; Fong et al. 2010 this work, and 3 ground-based measurements: Fong et al. 2012 Margutti et al. 2012 Sakamoto et al. 2013, Berger et al. 2013). For five bursts with no spectroscopic redshifts (GRBs 060121, 070707, 080503, 090305A, and 111020A), we have assumed $z=1$. Also shown are the cumulative distributions for long GRBs (black; Bloom et al. 2002), core-collapse SNe (green; Prieto et al. 2008), Type Ia SNe (blue; Prieto et al. 2008), and predicted offsets for NSNS binaries (grey; Fryer et al. 1999 , Bloom et al. 1999, Belczynski et al. 2006).

physical offsets, assuming $z \approx 1$ for bursts without known redshift. We find a range of $\approx 0.5-75 \mathrm{kpc}$ (Figure 6).

We supplement this sample of offsets with six measurements from Fong et al. (2010). In addition, we use offset measurements from ground-based observations of all of the remaining short GRBs with sub-arcsecond positions: GRB $111020 \mathrm{~A}$ with $6 \pm 1 \mathrm{kpc}$ (assuming $z \approx 1$, Fong et al. 2012), GRB 111117A with $10.5 \pm 1.7 \mathrm{kpc}$ (Margutti et al. 2012; Sakamoto et al. 2013), and GRB 120804A with $2.2 \pm$ $1.2 \mathrm{kpc}$ (Berger et al.2013). Therefore, the full sample of offsets includes 22 short GRBs (Figure 5) with a resulting median offset of $4.5 \mathrm{kpc}$. In comparison to the long GRB median offset of $1.3 \mathrm{kpc}$, the short GRB median offset is $\approx 3.5$ times larger. The short GRB median offset is comparable to those for Type Ia and core-collapse $\mathrm{SNe}$ of $\approx 3 \mathrm{kpc}$ (Figure 5. Prieto et al. 2008), but the short GRB offset distribution extends to much larger offsets: only $10 \%$ of both SN types have offsets of $\gtrsim 10 \mathrm{kpc}$, compared to $25 \%$ for short GRBs. Furthermore, no $\mathrm{SNe}$ have offsets of $\gtrsim 20 \mathrm{kpc}$, while $10 \%$ of short GRBs do.

In Figure 5, we also show a comparison of the short GRB offset distribution to the predicted distributions from population synthesis models of NS-NS binary mergers in Milky Way type galaxies (Fryer et al. 1999; Bloom et al. 1999; Belczynski et al. 2006). The short GRB distribution is broadly consistent with the NS-NS binary merger predictions, and is in very good agreement with two of the three models (Bloom et al. 1999, Belczynski et al. 2006). The median offset for the predicted distributions is $5-7 \mathrm{kpc}$, slightly larger than the observed median of $4.5 \mathrm{kpc}$. We note that the observed distribution is mainly derived from short GRBs with optical afterglows and may be missing a few bursts with less precise localization from X-ray afterglows (Fong et al.2013) that may occur outside of their host galaxies. Thus, while the observed distribution of offsets should be fairly representative of the true distribution, accounting for such missing events would 

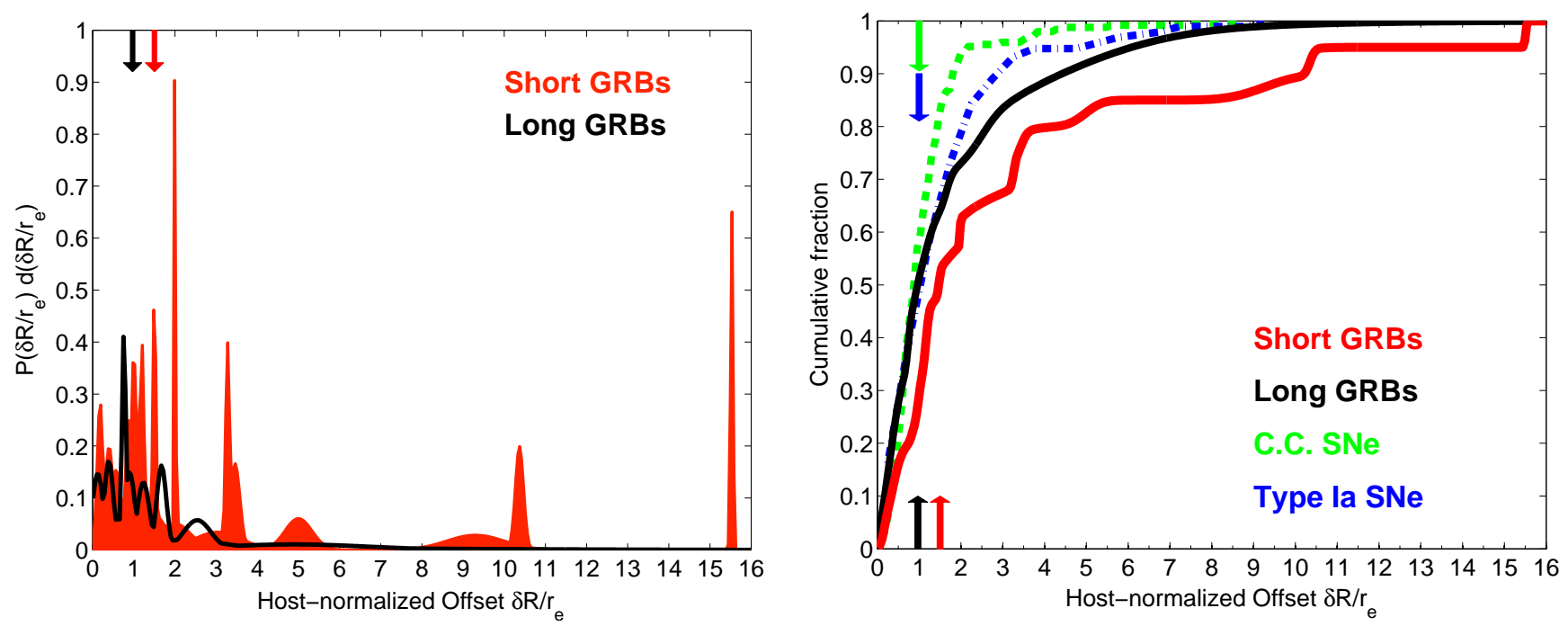

Figure 6. Left: Differential distributions of host-normalized offsets in units of effective radius, $r_{e}$, accounting for the uncertainty in each offset measurement, for short GRBs (red shaded region) and long GRBs (black line). The sample is comprised of 20 short GRBs with resolved host galaxies from HST data (Fong et al. 2010 and this work), including GRB 050509B which has only an XRT position. Arrows denote the weighted median offset for each population: 1.0 $r_{e}$ (long) and 1.5re (short). Right: Cumulative host-normalized offset distributions for short GRBs (red) and long GRBs (black). Also shown are the distributions for core-collapse supernovae (green dashed; Kelly \& Kirshner 2012) and Type Ia supernovae (blue dot-dashed; Galbany et al.2012). Arrows denote the weighted median offset for each population; the median for SNe is also $\approx 1 r_{e}$.

only extend the distribution to larger offsets, in even better agreement with the NS-NS merger models.

A study by Troja et al. (2008) suggested that short GRBs with extended emission in the X-rays have smaller offsets than short GRBs with no such emission. Two bursts in our sample, GRBs 070714B and 080503 have reported evidence for extended emission at $\gtrsim 5 \sigma$ significance (Racusin et al. 2007; Mao et al. 2008, Perley et al. 2009). GRB 070714B has an offset of $\approx 12.2 \mathrm{kpc}$ while GRB 080503 has an offset of $\approx 7.2 \mathrm{kpc}$ from its most probable host assuming $z \approx 1$. Combining these two bursts with four bursts analyzed in Fong et al. (2010) with sub-arcsecond positions and extended emission (GRBs 050709, 050724, 061006, and 060121), the median offset for the population is $3.2 \mathrm{kpc}$, with a range of $\approx 1-12 \mathrm{kpc}$. For the remaining 16 bursts with no extended emission and precise offset measurements, the median offset is $5.3 \mathrm{kpc}$. A Kolmogorov-Smirnov (K-S) test comparing the two populations gives a $p$-value of 0.9 , supporting the null hypothesis that the two populations are drawn from the same underlying distribution. Therefore, there is no clear evidence from their locations that short GRBs with and without extended emission require different progenitor systems.

To compare the offset distributions in a more uniform manner, we calculate host-normalized offsets, $\delta R / r_{e}$, using the effective radii as determined from our morphological analysis (Section 2.6). We find a range of host-normalized offsets of $\approx 0.3-15.5 r_{e}$ for the bursts with sub-arcsecond positions (Table 2). We supplement this sample with seven measurements from Fong et al. (2010), one of which has only an XRT position and thus a more uncertain offset (GRB 050509B). To account for the varying uncertainty in each offset, we plot a differential distribution of host-normalized offsets following the methodology of Bloom et al. (2002), as well as the resulting cumulative distribution (Figure 6). The total sample of short GRBs with host-normalized offsets is comprised of 20 events, with a median of $\approx 1.5 r_{e}$ and only about $25 \%$ of the events at $\lesssim 1 r_{e}$. For comparison, the host-normalized offset distributions for long GRBs (Fruchter et al. 2006), corecollapse SNe (Kelly et al. 2008) and Type Ia SNe (Galbany et al. 2012) have median offsets of $\approx 1 r_{e}$. Furthermore, a K-S test comparing the host-normalized offsets for long and short GRBs does not support the null hypothesis that the two populations are drawn from the same underlying distribution $(p=0.03)$. A K-S test between short GRBs and Type Ia SNe yields $p=10^{-3}$, indicating that the two populations are drawn from different host-normalized offset distributions. Indeed, $\approx 20 \%$ of short GRBs have offsets of $\gtrsim 5 r_{e}$, compared to only $\approx 5 \%$ for Type Ia SNe.

\subsection{Light Fraction}

To further study the local explosion environments of short GRBs, we utilize the fractional flux method which, unlike the spatial offset method, is independent of host morphology. We divide the fractional flux values into two categories based on the bursts' observed filters and redshifts: rest-frame UV $\left(\lambda_{\text {rest }} \lesssim 0.4 \mu \mathrm{m}\right)$ tracking star formation activity and restframe optical $\left(\lambda_{\text {rest }} \gtrsim 0.4 \mu \mathrm{m}\right)$ tracking stellar mass. For bursts with no redshift, we assume fiducial values of $z=1$ to determine the proper rest-frame band. For the 14 bursts in this analysis, all have rest-frame optical measurements while only three have rest-frame UV measurements (GRB 070707 assuming $z=1$, GRBs 070714B and 071227; Table 4). Despite having coincident host galaxies, these three bursts are located on the lowest level of their hosts' UV light with fractional flux measurements of zero. The rest-frame optical measurements span a range of $0-0.8$, with GRB 090426 as the highest measurement (Table 4).

We supplement these data with six additional bursts analyzed in Fong et al. (2010), bringing the total sample size to 20 events. We find that the resulting distributions are strongly skewed to low fractional flux measurements: $45 \%$ of short GRBs are located on the lowest optical brightness level of their hosts (fractional flux $\approx 0$ ), and $55 \%$ are on the lowest UV level (Figure 7 and Table 5). The short GRB distributions have very low median fractional flux values of $\approx 0.15$ for the optical and zero for the UV (Table 5 ). Furthermore, $\approx 75 \%$ of the events are located on the faint end (fractional flux $\lesssim 0.5$ ) of their hosts' optical and UV regions (Figure 7). A K-S test 
Table 5

Fractional Flux Statistics

\begin{tabular}{|c|c|c|c|c|c|c|c|}
\hline \multirow[b]{2}{*}{ Sample } & \multirow[b]{2}{*}{ Band } & \multirow[b]{2}{*}{ Median } & \multirow[b]{2}{*}{ Percentage at zero $^{a}$} & \multicolumn{4}{|c|}{$\mathrm{K}-\mathrm{S}$ test $p$-values } \\
\hline & & & & Linear $^{b}$ & Long GRBs & C. C. $\mathrm{SNe}$ & Type Ia SNe \\
\hline Short GRBs (all) ${ }^{c}$ & Optical & 0.15 & 45 & 0.04 & $\ldots$ & $\ldots$ & 0.02 \\
\hline Short GRBs $\left(P_{c c}(<\delta R) \lesssim 0.01\right)$ & Optical & 0.25 & 29 & 0.07 & $\ldots$ & $\ldots$ & 0.16 \\
\hline Short GRBs & UV & 0 & 55 & 0.01 & 0.002 & 0.002 & 0.34 \\
\hline Long GRBs & UV & 0.83 & 6 & 0.01 & $\ldots$ & 0.001 & $3.8 \times 10^{-4}$ \\
\hline C. C. $\mathrm{SNe}$ & UV & 0.60 & 5 & 0.42 & 0.001 & $\ldots$ & 0.04 \\
\hline Type Ia SNe & Optical & 0.34 & 6 & 0.35 & $\ldots$ & $\cdots$ & $\ldots$ \\
\hline Type Ia SNe & UV & 0.35 & 34 & 0.08 & $3.8 \times 10^{-4}$ & 0.04 & $\ldots$ \\
\hline
\end{tabular}

Note. - ${ }^{a}$ Percentage of a given population with fractional flux values of zero.

${ }^{b}$ Corresponds to a distribution that linearly tracks host galaxy light.

${ }^{c}$ All 20 short GRBs with sub-arcsecond positions.
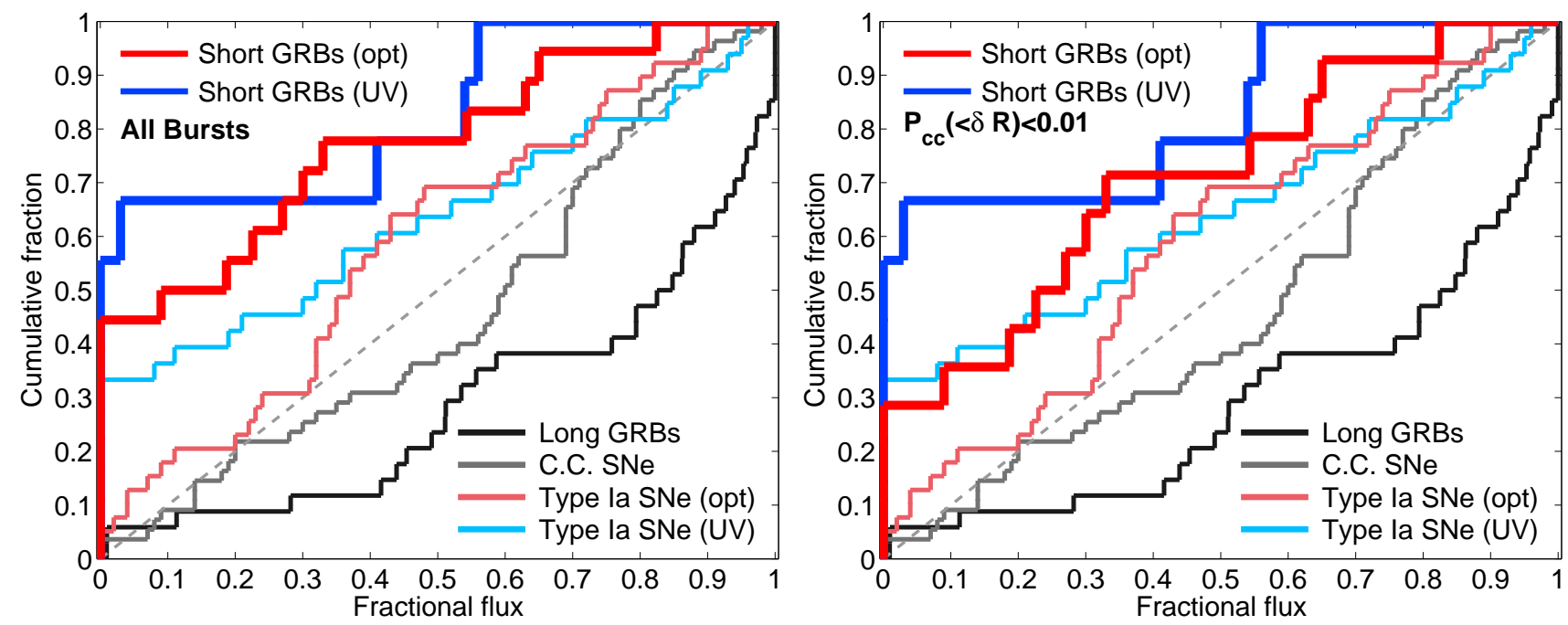

Figure 7. Left: Cumulative distribution of the fractional flux at short GRB locations relative to their host light. The rest-frame optical (0.4-1.2 $\mu$ m; red) and rest-frame UV ( $<0.4 \mu \mathrm{m}$; blue) for 20 short GRBs with $H S T$ observations are shown (Fong et al. 2010 and Table 4 in this work). For the purpose of determining the rest-frame band, bursts without known redshifts are assigned $z=1$. Also shown are the distributions for "normal-velocity" Type Ia supernovae for $u^{\prime}$-band (light blue) and $r^{\prime}$-band (pink; Wang et al. 2013), core-collapse supernovae (grey; Svensson et al. 2010) and long GRBs (black; Fruchter et al. 2006, Svensson et al. 2010). Right: Cumulative distribution of fractional flux including only bursts with host associations with $P_{c c}(<\delta R) \lesssim 0.01$. This case excludes four bursts: GRBs 061201, 080503, 080905A and 090515.

comparing the observed short GRB distribution to a distribution that is linearly correlated with host galaxy light (diagonal line in Figure 7) yields $p$-values of 0.04 and 0.01 for the optical and UV, respectively (Table 5). These results demonstrate that short GRBs are not correlated with their hosts' rest-frame UV and optical light, i.e., they do not trace regions of star formation or even stellar mass.

The short GRB distribution is particularly striking when compared to long GRBs, which lie on the brightest UV regions of their host galaxies and have a median fractional flux of 0.83 (Figure 7) and Table 5, Fruchter et al. 2006; Svensson et al. 2010). Core-collapse $\mathrm{SNe}$, which have a median value of 0.60 , may slightly over-represent their hosts' UV light (Svensson et al. 2010), commensurate with their origin in star-forming regions. Furthermore, at most a few percent of long GRBs and core-collapse $\mathrm{SNe}$ lie on the faintest regions of their host galaxies (Table 5).

On the other hand, Type Ia $\mathrm{SNe}$, which result from older stellar progenitor systems, slightly under-represent their hosts' UV and optical light distributions, although K-S test results indicate that this is only marginal (Table 5). In particular, $34 \%$ of Type Ia SNe have UV fractional flux values of zero, compared to $55 \%$ for short GRBs. The difference is more apparent in the optical, with only $6 \%$ of the Type Ia SNe population located on the faintest regions of their hosts, compared to $45 \%$ for short GRBs (Table 5). A K-S test comparing the distributions of short GRBs and Type Ia SNe indicates that the populations are not drawn from the same underlying distribution in the optical $(p=0.02)$.

We cannot completely rule out the remote possibility that the events with less robust host associations $\left(P_{c c}(<\delta R) \gtrsim\right.$ $0.01)$ and fractional flux values of zero instead originate from faint host galaxies with $m_{\mathrm{F} 160 \mathrm{~W}} \gtrsim 26.2 \mathrm{mag}$, below the detection threshold of the HST images. In this scenario, the fractional flux values for these events may be greater than zero. Therefore, if we only include events with $P_{c c}(<\delta R)<$ 0.01 (thereby excluding GRBs 061201, 080503, 080905A and 090515 from the distribution), we find that the short GRBs are still uncorrelated with their hosts' optical light ( $p=0.07$; Table 5), with a median value of $\approx 0.25$ (Figure 7 ). It is important to note that even in this conservative case, $\approx 30 \%$ of the bursts lie on the lowest optical flux levels of their hosts (Figure 7). The short GRB UV distribution is unaffected since there are no excluded bursts with rest-frame UV measure- 
ments.

\section{IMPLICATIONS FOR THE PROGENITORS}

Using the host associations, morphologies, offset distributions, and light distributions presented in the previous sections, we draw implications about the progenitor systems of short GRBs. We first note that the morphological analysis for the 16 host galaxies presented in this paper continues to support the apparent dominance of late-type host galaxies (starforming with disk morphologies), with only $\approx 1 / 4$ of short GRBs in hosts with elliptical morphologies (Berger 2009; Fong et al. 2010, 2013). The tendency for short GRBs to occur in star-forming galaxies indicates that the rate of short GRBs is driven by both recent star formation activity and stellar mass, as also inferred from the distribution of host galaxy masses (Leibler \& Berger 2010). In addition, the effective sizes of short GRB hosts are significantly larger than those for long GRB hosts, consistent with their larger luminosities, stellar masses, and metallicities (Berger 2009; Leibler \& Berger 2010).

In terms of locations relative to the host centers, we find that short GRBs span a wide range of projected physical offsets of $\sim 0.5-75 \mathrm{kpc}$, with a median value of about $4.5 \mathrm{kpc}$ and with about $25 \%$ of all events occurring at $\gtrsim 10 \mathrm{kpc}$. The median offset is 3.5 times larger than for long GRBs (Bloom et al. 2002, Fong et al. 2010). The larger offsets of short GRBs are also evident when normalizing by the effective radii of their hosts, with $\delta R / r_{e} \approx 0.3-16$ and a median value of $\delta R / r_{e} \approx 1.5$. In addition, only $25 \%$ of short GRBs have offsets of $\lesssim r_{e}$. The median value is 1.5 times larger than for long GRBs, core-collapse SNe, and even Type Ia SNe, which have $\left\langle\delta R / r_{e}\right\rangle \approx 1$. The broader distribution relative to long GRBs and SNe, and the fact that only about $20 \%$ of short GRBs occur within the radius that contains half the light indicate that short GRB progenitors migrate from their birth-sites before producing the bursts. Taken together with the overall match to population synthesis predictions (Figure 5), the physical and host-normalized offsets point to compact object binary progenitors with significant kicks.

In this context, the observed offset distribution depends on the combined distributions of kick velocities, merger timescales, and host galaxy masses. Therefore, the observed offset distribution, combined with estimates of the host galaxy stellar masses and merger timescales (Leibler \& Berger 2010) can provide insight into the kick velocity distribution. Considering the host stellar population ages as a proxy for the merger timescale, we expect the bursts with the largest offsets to originate from elliptical galaxies, since these systems have had the most time to travel prior to merger. However, the offsets are also sensitive to the escape velocities, and thus stellar masses, of the hosts, and we therefore expect short GRBs in low-mass galaxies to have larger offsets (Rosswog et al. 2003, Belczynski et al. 2006, Zemp et al. 2009). We investigate these effects using the host-normalized offsets from this work and Fong et al. (2010) in conjuncion with inferred stellar masses and stellar population ages from Leibler \& Berger (2010). In Figure 8 we plot the values of $\delta R / r_{e}$ versus stellar mass and population age for both early- and late-type hosts. We calculate the Kendall $\tau$ coefficient, (where a value of $\tau=1$ indicates statistical correlation), to assess whether the stellar population properties are correlated with host-normalized offsets, discarding the offset upper limits. We find that $\tau \approx 0.34$ ( $p=0.09)$ for stellar mass and $\tau \approx 0.26(p=0.20)$ for stellar population age. Both results agree with the null hypoth- esis that there is no strong correlation, and thus we find no clear trend between stellar population properties and offsets. In particular, we find that short GRBs in both elliptical and star-forming galaxies span the full range of host-normalized offsets. This result suggests that the observed offset distribution is primarily determined by the distribution of kick velocities.

Using the projected physical offsets and the stellar population ages as a proxy for the merger timescale we can calculate the minimum projected kick velocities if the progenitors originate at the host centers $\left(v_{\text {kick,min }}\right)$. We find a range of $v_{\text {kick,min }} \approx 2-150 \mathrm{~km} \mathrm{~s}^{-1}$, with a median of about $16 \mathrm{~km}$ $\mathrm{s}^{-1}$. However, a more reasonable value for the kick velocity of each system needs to take into account the host velocity dispersion $\left(v_{\text {disp }}\right)$, and we therefore use the geometric mean, $\sqrt{v_{\text {kick,min }} v_{\text {disp }}}$ (c.f., Bloom et al. 2007). Using a fiducial value for the late-type hosts of $v_{\text {disp }} \approx 120 \mathrm{~km} \mathrm{~s}^{-1}$, as measured for the Milky Way (Battaglia et al. 2005; Xue et al. 2008), and $\approx 250 \mathrm{~km} \mathrm{~s}^{-1}$ inferred for $\sim 10^{11} \mathrm{M}_{\odot}$ elliptical galaxies (Forbes \& Ponman 1999), we find projected kick velocities of $\approx 20-140 \mathrm{~km} \mathrm{~s}^{-1}$ with a median of $\approx 60 \mathrm{~km} \mathrm{~s}^{-1}$. This range is consistent with the inferred natal kick velocities for the eight known Galactic NS-NS binaries, which range from $\approx 5-500 \mathrm{~km} \mathrm{~s}^{-1}$ (Fryer \& Kalogera 1997, Wang et al. 2006, Wong et al. 2010).

Independent of the offset distribution, the locations of short GRBs relative to their hosts' light distribution also point to explosion sites that are distinct from the progenitor birth sites. In particular, our analysis clearly demonstrates that short GRBs are not spatially correlated with either star forming regions or even with the underlying distribution of stellar mass. This is unlike long GRBs and core-collapse SNe, which track UV light (Fruchter et al. 2006, Svensson et al. 2010), or Type Ia $\mathrm{SNe}$, which generally track stellar mass (Kelly et al. 2008). Most strikingly, about $30-45 \%$ of all short GRBs occur in regions that effectively contain no rest-frame optical light, and hence negligible stellar mass (Figure 7), indicating that the progenitors were not formed at the explosion sites. Moreover, studies of the host galaxy demographics and stellar mass distribution show that the short GRB rate depends on both stellar mass and star formation activity (Leibler \& Berger 2010, Fong et al. 2013), while the light distributions point to explosion sites that are de-coupled from both star-forming regions and the stellar mass distribution. The combination of these results, along with the large host-normalized offsets, provides the strongest support to date for NS-NS/NS-BH progenitors with significant migration from their birth sites to their eventual explosion sites.

Finally, the $H S T$ observations presented here provide unprecedented NIR limits on coincident hosts for short GRBs previously termed as host-less based on ground-based optical data (GRBs 061201, 070809, 080503 and 090515). Using these limits we investigate the possibility that these events are not at large offsets from their hosts (as appears to be the case based on probability of chance coincidence arguments; Berger 2010 and this paper), but instead originate from coincident hosts that are below the HST detection limit.

To determine the combination of luminosity and redshift required for such faint coincident hosts, we compare the average $3 \sigma$ limit of the $H S T$ observation, $m_{160 \mathrm{w}} \gtrsim 26.2 \mathrm{mag}$, to the observer-frame $H$-band galaxy luminosity function taking into account its evolution with redshift (Figure 9). We find that if these hosts are $\sim L^{*}$ galaxies, typical of other short 

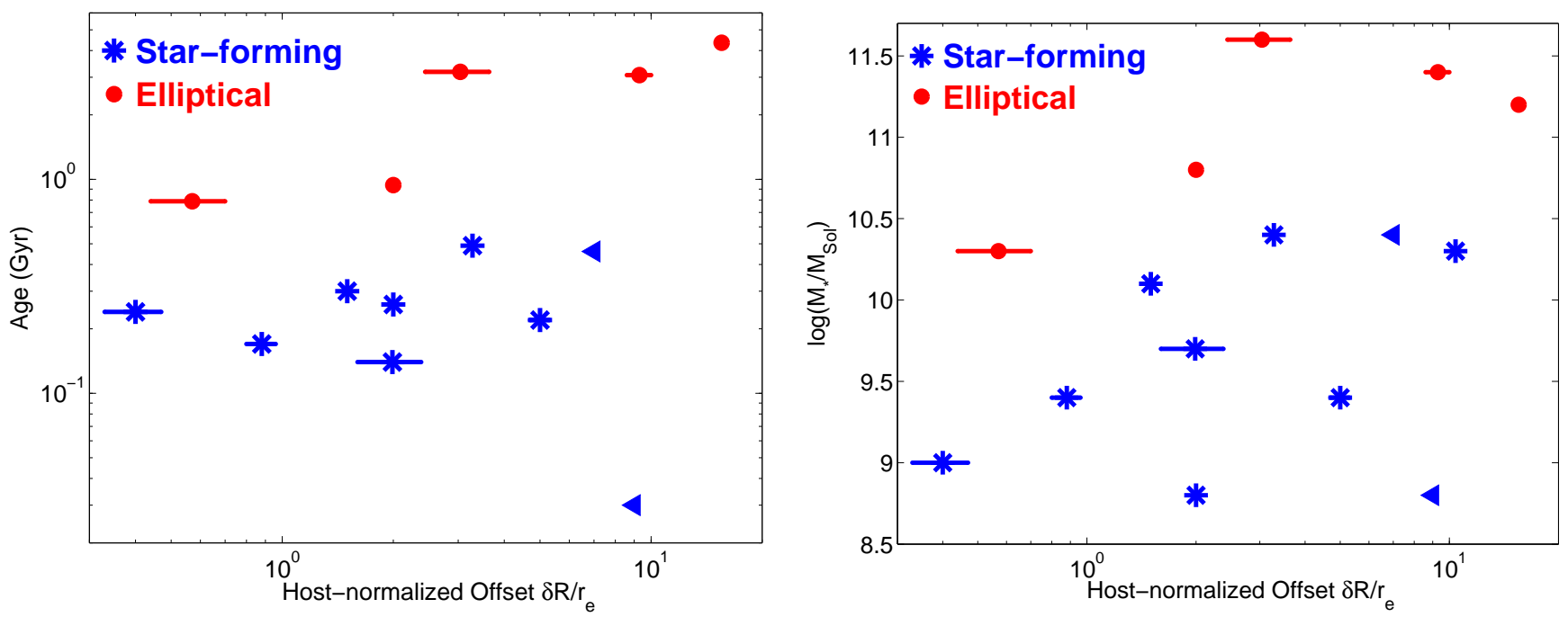

Figure 8. Stellar population age (left) and stellar mass (right) versus host-normalized offsets for 15 short GRB host galaxies (Leibler \& Berger 2010 Rowlinson et al. 2010b), including three bursts with only XRT positions (GRBs 050509B, 051210 and 070429B). Star-forming (blue stars) and elliptical hosts (red circles), as determined from spectroscopy, are indicated. Triangles denote bursts with no detected optical afterglow but that have a single galaxy within the XRT error circle and therefore an upper limit on the offset. We find no obvious trends between stellar mass and host-normalized offsets, or between stellar population age and host-normalized offsets.

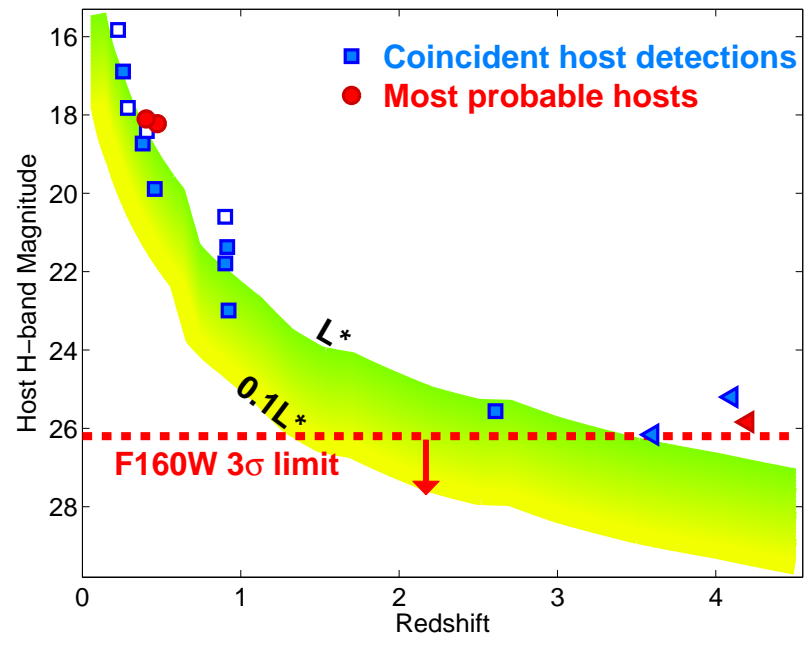

Figure 9. $H$-band host galaxy apparent magnitudes from ground-based and $H S T$ observations versus redshift for short GRBs with coincident host galaxies (blue squares and triangles) and the most probable hosts of GRBs 070809, 080503 and 090515 (red circles and triangle). Triangles denote upper limits on the burst redshift from the detection of the optical afterglow in a particular band. Open symbols correspond to bursts with XRT positions only and a probable host galaxy (GRBs 050509B, 060502B, 070429B and 100206A; Bloom et al. 2007 Perley et al. 2012). The average $3 \sigma$ upper limit of $m_{\mathrm{F} 160 \mathrm{~W}} \approx 26.2 \mathrm{mag}$ for the bursts lacking coincident host galaxies (dotted red line) and the evolving luminosity function for $0.1 L^{*}$ to $L^{*}$ galaxies (green shaded area; Chen et al. 2003, Poli et al. 2003, Saracco et al. 2006, Marchesini et al. 2007, Hill et al. 2010, Ramos et al. 2011. Marchesini et al. 2012 Stefanon \& Marchesini 2013) are shown.

GRB hosts (Figure 9, Berger 2009), they would need to originate at $z \gtrsim 3.5$. The highest known short GRB redshift is $z=2.609$ (GRB 090426), while typical redshifts are $\sim 0.2-1$, so in this scenario, these bursts would represent a distinct population of the highest redshift short GRBs. If instead the bursts have redshifts following the observed redshift distribution, this would require the hosts to have luminosities well below $0.1 L^{*}$ (Figure 9), at least an order of magnitude below the typical luminosities of short GRB hosts. Furthermore, Berger (2010) show that these bursts have systematically fainter optical afterglows than bursts with coincident hosts, which is at odds with the scenario of similar redshifts and sub-luminous hosts. Thus, we do not consider the possibility that the hostless events have coincident hosts below the detection limit of the HST data as likely. Instead, when combined with a probability of chance coincidence analysis (Berger 2010 and this paper), these bursts appear to be associated with galaxies that are typical of the short GRB host population (Figure 9), with resulting offsets of $\sim 10-100 \mathrm{kpc}$. Thus, the deep NIR limits presented here provide further evidence for large offsets consistent with NS-NS/NS-BH progenitors.

\section{CONCLUSIONS}

We presented HST observations and a detailed analysis of 22 short GRB host galaxies. Based on this analysis combined with the results from Fong et al. 2010, we draw several key conclusions:

1. Short GRB host galaxies with disk morphologies dominate the sample, with only $\approx 1 / 4$ of the hosts having elliptical morphologies. The median effective size of short GRB hosts is $\approx 3.6 \mathrm{kpc}$, about twice as large as long GRB hosts, which are exclusively associated with late-type, star-forming galaxies.

2. Short GRBs have projected physical offsets from their host galaxies of $\approx 0.5-75 \mathrm{kpc}$, with a median of $\approx 4.5 \mathrm{kpc}, 3.5$ times larger than the offsets for long GRBs. Compared to the distributions for core-collapse and Type Ia SNe, short GRBs extend to larger offsets, with $\approx 25 \%$ of events at $\gtrsim 10 \mathrm{kpc}$, compared to only $10 \%$ for both $\mathrm{SN}$ types.

3. Taking into account their host sizes, short GRBs have host-normalized offsets of $0.3-15.5 r_{e}$ with a median of $\approx 1.5 r_{e}$, approximately 1.5 times larger than those for long GRBs, core-collapse SNe, and Type Ia SNe. Furthermore, $\approx 20 \%$ of short GRBs have offsets of $\gtrsim$ $5 r_{e}$, compared to only $5 \%$ for Type Ia $\mathrm{SNe}$, which also result from old stellar progenitors.

4. In the context of NS-NS/NS-BH progenitors, we use the offset distribution, stellar population age distribution, and typical velocity dispersions for star-forming 
and elliptical galaxies, to infer kick velocities of $\approx$ $20-140 \mathrm{~km} \mathrm{~s}^{-1}$ with a median of $\approx 60 \mathrm{~km} \mathrm{~s}^{-1}$. This is generally consistent with the range of kick velocities inferred for Galactic NS-NS binaries.

5. Short GRBs severely under-represent their hosts' restframe UV or optical light. In particular, $30-45 \%$ of short GRBs are located on the faintest optical regions of their host galaxies, while $\approx 55 \%$ occur in the faintest UV regions, showing that short GRBs do not spatially track star formation or stellar mass. Combined with the host galaxy demographics which imply a short GRB rate driven by both star formation and stellar mass, this demonstrates that short GRBs migrate from their birth sites to their eventual explosion sites and provides strong support for progenitor kicks, ie., NS-NS/NS-BH mergers.

6. For bursts with no robust association to a host galaxy within $\sim$ few arcsec, we consider a faint coincident host origin by comparing the NIR limit of $m_{\mathrm{F} 160 \mathrm{~W}} \gtrsim$ $26.2 \mathrm{mag}$ to the $H$-band galaxy luminosity function and other short GRB hosts. If these hosts are $\sim L^{*}$ galaxies, typical of other short GRB hosts, they are constrained to $z \gtrsim 3.5$. Alternatively, if these bursts occur at typical short GRB redshifts of $z \sim 0.5$, this requires sub-luminous hosts compared to the population, with $\lesssim 0.1 L^{*}$. Instead, it is more likely that these bursts originate from $\sim L^{*}$ galaxies at $\approx 10-100 \mathrm{kpc}$ offsets as indicated by probability of chance coincidence analysis.

Through this analysis, we have provided independent lines of evidence which argue for NS-NS/NS-BH mergers as the progenitors of short GRBs. In particular, both the spatial offsets and their locations on the faintest regions of their hosts demonstrate that the progenitors must migrate between their formation and the eventual explosions. In addition, we have made detailed comparisons between short GRBs and Type Ia SNe which, unlike long GRBs and core-collapse SNe, result from old stellar progenitors. We find that the two populations differ in their distributions of host-normalized offsets and restframe optical light locations, with short GRBs having larger offsets and a weaker correlation with stellar mass. Finally, we note that the large fraction of short GRBs with a weak correlation to stellar light agrees with the overall indication from the afterglow emission that the parsec-scale densities around the progenitors are generally low, $\lesssim 0.1 \mathrm{~cm}^{-3}$ (Soderberg et al. 2006).

The Berger GRB group is supported by the National Science Foundation under Grant AST-1107973, and previously by NASA/Swift AO6 grant NNX10AI24G and A07 grant NNX12AD69G. This paper includes data gathered with the 6.5 meter Magellan Telescopes located at Las Campanas Observatory, Chile. This work is based in part on observations obtained at the Gemini Observatory, which is operated by the Association of Universities for Research in Astronomy, Inc., under a cooperative agreement with the NSF on behalf of the Gemini partnership: the National Science Foundation (United States), the Science and Technology Facilities Council (United Kingdom), the National Research Council (Canada), CONICYT (Chile), the Australian Research Council (Australia), Ministério da Ciência, Tecnologia e Inovação
(Brazil) and Ministerio de Ciencia, Tecnología e Innovación Productiva (Argentina). This work made use of data supplied by the UK Swift Science Data Centre at the University of Leicester. Based on observations made with ESO Telescopes at the La Silla Paranal Observatory under programme ID 59.A-9002(D), 079.D-0909(C), 080.D-0906(G), 081.D-0588(C), 083.D-0606(C), and 084.D-0621(B). Based on observations made with the NASA/ESA Hubble Space Telescope, obtained from the data archive at the Space Telescope Science Institute. STScI is operated by the Association of Universities for Research in Astronomy, Inc. under NASA contract NAS 5-26555. 


\section{REFERENCES}

Alard, C. 2000, A\&AS, 144, 363

Antonelli, L. A., et al. 2009a, A\&A, 507, L45

Antonelli, L. A., et al. 2009b, A\&A, 507, L45

Battaglia, G., et al. 2005, MNRAS, 364, 433

Belczynski, K., Perna, R., Bulik, T., Kalogera, V., Ivanova, N., \& Lamb, D. Q. 2006, ApJ, 648, 1110

Berger, E. 2009, ApJ, 690, 231

Berger, E. 2010, ApJ, 722, 1946

Berger, E., Cenko, S. B., Fox, D. B., \& Cucchiara, A. 2009, ApJ, 704, 877

Berger, E., Fong, W., \& Chornock, R. 2013, ArXiv e-prints

Berger, E., et al. 2013, ApJ, 765, 121

Bloom, J. S., Kulkarni, S. R., \& Djorgovski, S. G. 2002, AJ, 123, 111

Bloom, J. S., et al. 2007, ApJ, 654, 878

Bloom, J. S., Sigurdsson, S., \& Pols, O. R. 1999, MNRAS, 305, 763

Cenko, S. B., et al. 2008, ArXiv e-prints

Chapman, R., Levan, A. J., Wynn, G. A., Davies, M. B., King, A. R.,

Priddey, R. S., \& Tanvir, N. R. 2008, in American Institute of Physics

Conference Series, Vol. 983, 40 Years of Pulsars: Millisecond Pulsars,

Magnetars and More, ed. C. Bassa, Z. Wang, A. Cumming, \&

V. M. Kaspi, 301

Chen, H.-W., et al. 2003, ApJ, 586, 745

Ciotti, L., \& Bertin, G. 1999, A\&A, 352, 447

Cucchiara, A., Prochaska, J. X., Perley, D. A., Cenko, S. B., Werk, J., Cao, Y., Bloom, J. S., \& Cobb, B. E. 2013, ArXiv e-prints

D’Avanzo, P., et al. 2009, A\&A, 498, 711

Eichler, D., Livio, M., Piran, T., \& Schramm, D. N. 1989, Nature, 340, 126

Evans, P. A., et al. 2009, MNRAS, 397, 1177

Fong, W., et al. 2013, ApJ, 769, 56

Fong, W., et al. 2011, ApJ, 730, 26

Fong, W., Berger, E., \& Fox, D. B. 2010, ApJ, 708, 9

Fong, W., et al. 2012, ApJ, 756, 189

Forbes, D. A., \& Ponman, T. J. 1999, MNRAS, 309, 623

Fruchter, A. S., et al. 2006, Nature, 441, 463

Fryer, C., \& Kalogera, V. 1997, ApJ, 489, 244

Fryer, C. L., Woosley, S. E., \& Hartmann, D. H. 1999, ApJ, 526, 152

Galbany, L., et al. 2012, ApJ, 755, 125

Goad, M. R., et al. 2007, A\&A, 476, 1401

Graham, J. F., et al. 2009, ApJ, 698, 1620

Hakobyan, A. A., Petrosian, A. R., McLean, B., Kunth, D., Allen, R. J., Turatto, M., \& Barbon, R. 2008, A\&A, 488, 523

Hill, D. T., Driver, S. P., Cameron, E., Cross, N., Liske, J., \& Robotham, A. 2010, MNRAS, 404, 1215

Holland, S. T., de Pasquale, M., \& Markwardt, C. B. 2007, GRB Coordinates Network, 7145, 1

Kelly, P. L., \& Kirshner, R. P. 2012, ApJ, 759, 107

Kelly, P. L., Kirshner, R. P., \& Pahre, M. 2008, ApJ, 687, 1201

Kocevski, D., et al. 2010, MNRAS, 404, 963

Kouveliotou, C., Meegan, C. A., Fishman, G. J., Bhat, N. P., Briggs, M. S., Koshut, T. M., Paciesas, W. S., \& Pendleton, G. N. 1993, ApJ, 413, L101

Landsman, W., Marshall, F. E., \& Racusin, J. 2007, GRB Coordinates Network, 6689, 1

Leibler, C. N., \& Berger, E. 2010, ApJ, 725, 1202

Levan, A. J., Tanvir, N. R., Hjorth, J., Malesani, D., de Ugarte Postigo, A., \& D’Avanzo, P. 2009, GRB Coordinates Network, 10154, 1
Levan, A. J., Wynn, G. A., Chapman, R., Davies, M. B., King, A. R., Priddey, R. S., \& Tanvir, N. R. 2006, MNRAS, 368, L1

Levesque, E. M., et al. 2010, MNRAS, 401, 963

Li, W., Chornock, R., Leaman, J., Filippenko, A. V., Poznanski, D., Wang, X., Ganeshalingam, M., \& Mannucci, F. 2011, MNRAS, 412, 1473

Mannucci, F., Della Valle, M., Panagia, N., Cappellaro, E., Cresci, G., Maiolino, R., Petrosian, A., \& Turatto, M. 2005, A\&A, 433, 807

Mao, J., Guidorzi, C., Ukwatta, T., Brown, P. J., Barthelmy, S. D., Burrows, D. N., Roming, P., \& Gehrels, N. 2008, GCN Report, 138, 1

Marchesini, D., Stefanon, M., Brammer, G. B., \& Whitaker, K. E. 2012, ApJ, 748, 126

Marchesini, D., et al. 2007, ApJ, 656, 42

Margutti, R., et al. 2012, ApJ, 756, 63

McBreen, S., et al. 2010, A\&A, 516, A71

Metzger, B. D., Quataert, E., \& Thompson, T. A. 2008, MNRAS, 385, 1455

Narayan, R., Paczynski, B., \& Piran, T. 1992, ApJ, 395, L83

Nicuesa Guelbenzu, A., et al. 2012, A\&A, 538, L7

Oemler, A., Jr., \& Tinsley, B. M. 1979, AJ, 84, 985

Perley, D. A., Bloom, J. S., Modjaz, M., Miller, A. A., Shiode, J., Brewer, J., Starr, D., \& Kennedy, R. 2008, GRB Coordinates Network, 7889, 1 Perley, D. A., et al. 2009, ApJ, 696, 1871

Perley, D. A., Modjaz, M., Morgan, A. N., Cenko, S. B., Bloom, J. S., Butler, N. R., Filippenko, A. V., \& Miller, A. A. 2012, ApJ, 758, 122

Perley, D. A., Thoene, C. C., Cooke, J., Bloom, J. S., \& Barton, E. 2007, GRB Coordinates Network, 6739, 1

Piranomonte, S., et al. 2008, A\&A, 491, 183

Poli, F., et al. 2003, ApJ, 593, L1

Prieto, J. L., Stanek, K. Z., \& Beacom, J. F. 2008, ApJ, 673, 999

Qin, B., Wu, X.-P., Chu, M.-C., Fang, L.-Z., \& Hu, J.-Y. 1998, ApJ, 494, L57

Racusin, J., Barbier, L., \& Landsman, W. 2007, GCN Report, 70, 1

Ramos, B. H. F., et al. 2011, AJ, 142, 41

Rosswog, S., Ramirez-Ruiz, E., \& Davies, M. B. 2003, MNRAS, 345, 1077

Rowlinson, A., et al. 2010a, MNRAS, 409, 531

Rowlinson, A., et al. 2010b, MNRAS, 408, 383

Sakamoto, T., et al. 2013, ApJ, 766, 41

Saracco, P., et al. 2006, MNRAS, 367, 349

Schlafly, E. F., \& Finkbeiner, D. P. 2011, ApJ, 737, 103

Soderberg, A. M., et al. 2006, ApJ, 650, 261

Stefanon, M., \& Marchesini, D. 2013, MNRAS, 429, 881

Stratta, G., et al. 2007, A\&A, 474, 827

Svensson, K. M., Levan, A. J., Tanvir, N. R., Fruchter, A. S., \& Strolger, L.-G. 2010, MNRAS, 405, 57

Tanvir, N. R., Levan, A. J., Fruchter, A. S., Hjorth, J., Wiersema, K., Tunnicliffe, R., \& de Ugarte Postigo, A. 2013, ArXiv e-prints

Troja, E., King, A. R., O’Brien, P. T., Lyons, N., \& Cusumano, G. 2008, MNRAS, 385, L10

van den Bergh, S., Li, W., \& Filippenko, A. V. 2005, PASP, 117, 773

Wainwright, C., Berger, E., \& Penprase, B. E. 2007, ApJ, 657, 367

Wang, C., Lai, D., \& Han, J. L. 2006, ApJ, 639, 1007

Wang, X., Wang, L., Filippenko, A. V., Zhang, T., \& Zhao, X. 2013, ArXiv e-prints

Wong, T.-W., Willems, B., \& Kalogera, V. 2010, ApJ, 721, 1689

Xue, X. X., et al. 2008, ApJ, 684, 1143

Zemp, M., Ramirez-Ruiz, E., \& Diemand, J. 2009, ApJ, 705, L186 